\title{
Visualizing protein breathing motions associated with aromatic ring flipping
}

https://doi.org/10.1038/s41586-022-04417-6

Received: 30 April 2021

Accepted: 7 January 2022

Published online: 16 February 2022

\section{Open access}

Check for updates

\author{
Laura Mariño Pérez ${ }^{1,2}$, Francesco S. lelasi ${ }^{3}$, Luiza M. Bessa', Damien Maurin', Jaka Kragelj, ${ }^{1,5}$, \\ Martin Blackledge', Nicola Salvi', Guillaume Bouvignies ${ }^{4}$, Andrés Palencia ${ }^{3 凶} \&$ \\ Malene Ringkjøbing Jensen ${ }^{1 凶}$
}

\begin{abstract}
Aromatic residues cluster in the core of folded proteins, where they stabilize the structure through multiple interactions. Nuclear magnetic resonance (NMR) studies in the 1970 s showed that aromatic side chains can undergo ring flips - that is, $180^{\circ}$ rotations-despite their role in maintaining the protein fold $^{1-3}$. It was suggested that large-scale 'breathing' motions of the surrounding protein environment would be necessary to accommodate these ring flipping events ${ }^{1}$. However, the structural details of these motions have remained unclear. Here we uncover the structural rearrangements that accompany ring flipping of a buried tyrosine residue in an $\mathrm{SH} 3$ domain. Using NMR, we show that the tyrosine side chain flips to a low-populated, minor state and, through a proteome-wide sequence analysis, we design mutants that stabilize this state, which allows us to capture its high-resolution structure by X-ray crystallography. A void volume is generated around the tyrosine ring during the structural transition between the major and minor state, and this allows fast flipping to take place. Our results provide structural insights into the protein breathing motions that are associated with ring flipping. More generally, our study has implications for protein design and structure prediction by showing how the local protein environment influences amino acid side chain conformations and vice versa.
\end{abstract}

Aromatic residues make up a considerable fraction of the hydrophobic core of folded proteins, where they stabilize the structure through $\mathrm{CH}-\pi$ (refs. ${ }^{4-6}$ ), $\Pi-\pi$ (refs. ${ }^{7,8}$ ) and cation $-\pi$ (refs. ${ }^{9,10}$ ) interactions as well as hydrogen bonds. NMR studies in the 1970 s demonstrated that aromatic side chains can undergo ring flips - that is, $180^{\circ}$ rotations of the $\chi_{2}$ dihedral angle $(C \beta-C \gamma$ axis $)$-even when engaged in stabilizing interactions in the hydrophobic core ${ }^{1-3}$. These ring flips require concerted movements of the surrounding residues (large-amplitude protein breathing motions), and ring flipping rates as a function of temperature and pressure have been used to report on these motions by deriving activation energies and volumes ${ }^{11-21}$. However, the structural details of ring flipping and the associated breathing motions have remained unknown, probably owing to difficulties in stabilizing ring flipping transition states or intermediates that are amenable to structure elucidation.

Here we capture ring flipping events of a buried tyrosine residue in the SH3 domain of the JNK-interacting protein 1 (JIP1). We show using NMR relaxation dispersion that the aromatic ring of this tyrosine residue populates a minor-state conformation (3\%), and we design single point mutations to stabilize this conformation and capture its high-resolution structure using X-ray crystallography. The structure reveals how the intricate network of hydrogen bonds and $\mathrm{CH}-\pi$ interactions is rearranged in the minor state. We show how a substantial void volume is generated around the tyrosine ring during the structural transition from the major to the minor state, which can be associated with the breathing motions that allow fast-timescale ring flipping events to take place. Our results provide structural insights into aromatic ring flipping and its associated protein breathing motions.

\section{Protein dynamics induced by a tyrosine residue}

The SH3 domain of JIP1 undergoes exchange between two distinct conformations, as evidenced by ${ }^{15} \mathrm{~N}$ NMR relaxation measured at multiple temperatures (Extended Data Figs. 1, 2, Supplementary Discussion). To analyse the observed dynamics in detail, we acquired ${ }^{15} \mathrm{~N}$ and ${ }^{1} \mathrm{H}^{\mathrm{N}}$ Carr-Purcell-Meiboom-Gill (CPMG) relaxation dispersion experiments at $15^{\circ} \mathrm{C}$ (refs. ${ }^{22-25}$ ). These experiments quantify the kinetics of exchange processes and provide the difference in chemical shift between a major and a minor state, together with their relative populations ${ }^{26-30}$. The data confirm that exchange contributions to the transverse relaxation are present for residues within three regions of the protein (Extended Data Fig. 1d, e, Supplementary Table 1). These residues are located spatially close to tyrosine 526 (Y526) (Extended Data Fig. 1f). A mutation of Y526 to alanine (Y526A) shows no conformational exchange (Extended Data Fig. 1d, e), and conserves the protein backbone conformation, as evidenced from its crystal structure that we obtained at $1.5-\AA$ A resolution (Extended

'Université Grenoble Alpes, CEA, CNRS, IBS, Grenoble, France. ${ }^{2}$ Departament de Química, Universitat de les Illes Balears, Palma de Mallorca, Spain. ${ }^{3}$ Institute for Advanced Biosciences (IAB),

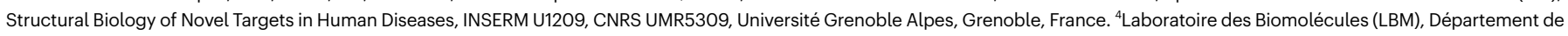
Chimie, École normale supérieure, PSL University, Sorbonne Université, CNRS, Paris, France. ${ }^{5}$ Present address: Department of Biophysics, University of Texas Southwestern Medical Center,

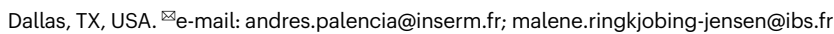



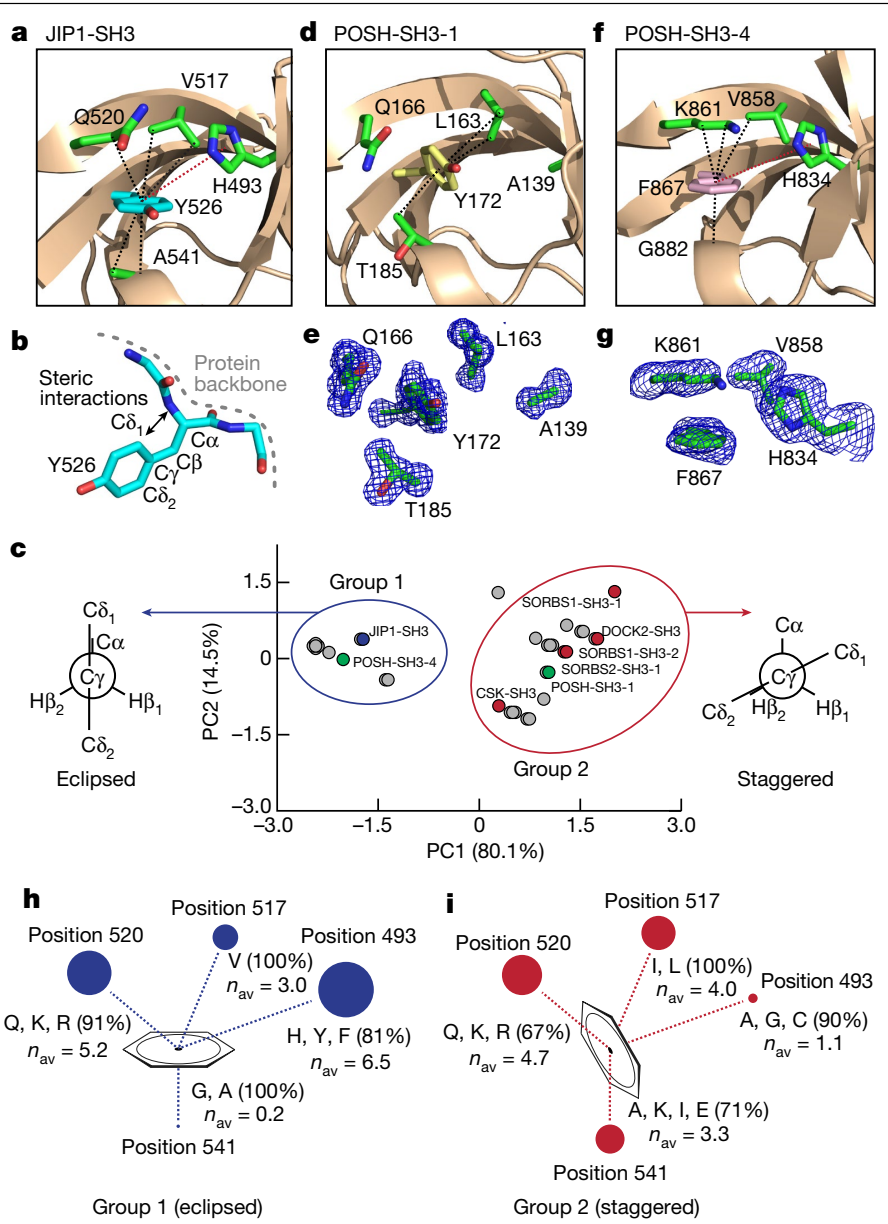

Fig. 1 The side chain conformation of Y526 is determined by steric interactions with the surrounding amino acids. a, Crystal structure of wild-type JIP1-SH3, showing the conformation of Y526 and its stabilizing interactions with H493, V517, Q520 and A541. Dashed lines indicate $\mathrm{CH}-\pi$ (black) and $\pi-\pi$ (red) interactions. b, Side chain conformation of Y526 in JIP1-SH3, illustrating the steric interactions between the $\delta_{1}$ nuclei of the aromatic ring and the backbone. $\mathbf{c}$, PCA of a dataset comprising the size of the amino acid side chains at positions 493, 517 and 541 within $\mathrm{SH} 3$ domains that contain $\mathrm{Y}$ or $\mathrm{F}$ at position 526 (Extended Data Fig. 5b). Two groups are observed, which correspond to eclipsed (group 1) or staggered (group 2) conformations of the aromatic ring. JIP1-SH3 is indicated in blue; $\mathrm{POSH}-\mathrm{SH} 3-1$ and $\mathrm{POSH}-\mathrm{SH} 3-4$ are indicated in green; and $\mathrm{SH} 3$ domains for which crystal structures have been determined previously are shown in red. d, Crystal structure of POSH-SH3-1, showing a staggered conformation of Y172. e, Unbiased electron density maps (Fo-Fc) of Y172 and the surrounding residues in POSH-SH3-1.f, Crystal structure of POSH-SH3-4, showing an eclipsed conformation of F867. g, Unbiased electron density maps (Fo-Fc) of F867 and the surrounding residues in POSH-SH3-4. h, i, Results of the PCA, illustrating the size and nature of the residues that surround the aromatic ring in position 526 in group 1 (h) and group 2 (i) $\mathrm{SH} 3$ domains. The size of the spheres in each position is proportional to the average size $\left(n_{\mathrm{av}}\right)$ of the amino acid side chain across group members.

Data Fig. 3, Extended Data Tables 1,2). These results show that the relaxation dispersion that affects around $40 \%$ of the residues in the $\mathrm{SH} 3$ domain arises from a single exchange process, with Y526 being the origin of the observed exchange.

The ${ }^{15} \mathrm{~N}$ and ${ }^{1} \mathrm{H}^{\mathrm{N}}$ relaxation dispersion data were analysed simultaneously according to a two-site exchange model in which a highly populated major state interconverts with a low-populated minor state (Extended Data Fig. 4a, b, Supplementary Discussion). The analysis of the data gives a population of the minor state of $p_{\text {minor }}=2.8 \pm 0.1 \%$ and an exchange rate constant of $k_{\mathrm{EX}}=2,600 \pm 70 \mathrm{~s}^{-1}$. The derived chemical shift differences, $\Delta \delta_{\mathrm{CPMG}}$, span a range of $4.7 \mathrm{ppm}$ for ${ }^{15} \mathrm{~N}$ and $1.1 \mathrm{ppm}$ for ${ }^{1} \mathrm{H}^{\mathrm{N}}$ suggesting that there are substantial structural changes between the major and the minor state (Extended Data Fig. 4c, d).

The side chain of Y526 is found in an unusual conformation in the crystal structure (Protein Data Bank (PDB) code 2FPE (ref. ${ }^{31}$ ), Extended Data Table 1$)$, characterized by a $\chi_{2}$ dihedral angle of $2^{\circ}$ (Fig. 1a). Normally, this eclipsed conformation is energetically unfavourable because of steric interactions with the backbone (Fig. 1b), and it is rarely found in proteins as $\chi_{2}$ angles are preferred where $C \delta_{1}$ and $C \delta_{2}$ are staggered with respect to $C \alpha\left(\right.$ ref. ${ }^{32}$ ). However, the eclipsed conformation of the aromatic ring of Y526 is stabilized by $\mathrm{CH}-\pi$ interactions from V517, Q520 and A541 and by $\pi-\pi$ interactions with H493 (Fig. 1a).

\section{Proteome-wide SH3 sequence analysis}

To investigate the contribution from the surrounding residues in stabilizing the eclipsed conformation of Y526, we analysed the sequences of all identified human $\mathrm{SH} 3$ domains ${ }^{33}$. We categorized the sequences according to the identity of the amino acid at the position of Y526 in the JIP1SH3 domain (JIP1-SH3) (Extended Data Fig. 5a), and we retained the sequences carrying a phenyl-based amino acid (Tyr or Phe) at this position, amounting to $33 \mathrm{SH} 3$ domains. Sequence alignments reveal a large variation in the size of the amino acids at positions 493, 517 and 541, whereas at position 520 most sequences contain Gln, Arg or Lys (72\%) (Extended Data Fig. 5b, c). To study the size correlation between the amino acids at positions 493, 517 and 541 and their influence on the conformation of the aromatic residue at position 526, we carried out a principal component analysis (PCA) by assigning a size score $(n)$ to each amino acid according to the number of heavy atoms in their side chains. This analysis reveals two well-separated groups, with the SH3 domain of JIP1 belonging to group 1 (Fig. 1c). In group 2, five SH3 domains are found for which high-resolution crystal structures are available; these include three $\mathrm{SH} 3$ domains of the sorbin and $\mathrm{SH} 3$ domain-containing proteins 1 and 2 (SORBS1 and SORBS2) ${ }^{34}$, and the SH3 domains of the dedicator of cytokinesis protein 2 (DOCK2) ${ }^{35}$ and of the tyrosine protein kinase $\mathrm{CSK}^{36}$ (Extended Data Fig. 5b). Notably, all group 2 structures show a favourable, staggered side chain conformation (of $\mathrm{C} \delta_{1} / \mathrm{C} \delta_{2}$ with respect to $\mathrm{C} \alpha$ ) of the corresponding tyrosine, with the $\chi_{2}$ dihedral angle ranging from $-40^{\circ}$ to $-64^{\circ}$ (Fig. 1c). We therefore hypothesized that $\mathrm{SH} 3$ domains of group 1 have eclipsed conformations, whereas group 2 have staggered conformations. To test our hypothesis, we determined two crystal structures of $\mathrm{SH} 3$ domains of the scaffold protein POSH ('plenty of SH3 domains'), for which the first SH3 domain belongs to group 2 and the fourth $\mathrm{SH} 3$ domain belongs to group 1 (Extended Data Table 2, Fig. 1c). Consistent with the PCA, the crystal structure of the first SH3 domain of POSH (POSH-SH3-1) shows a staggered conformation of the corresponding tyrosine residue (Y172), which is stabilized by $\mathrm{CH}-\pi$ interactions from $\mathrm{L} 163$ (position 517) and T185 (position 541) (Fig. 1d, e). POSH-SH3-4 shows a similar structure to JIP1-SH3, with an eclipsed conformation of the corresponding phenylalanine residue ( $\mathrm{F} 867$ ) stabilized by $\mathrm{CH}-\pi$ interactions from V858 (position 517), K861 (position 520) and G882 (position 541), and by $\pi-\pi$ interactions with H834 (position 493) (Fig. 1f, g). Our data therefore suggest that the conformation of the aromatic ring at position 526 is determined by steric interactions dictated by the size of the surrounding amino acids.

\section{Structure of the minor state}

Next, we investigated whether the minor state detected by NMR corresponds to a staggered conformation of the side chain of Y526. We sought to stabilize the minor state relative to the major state by 


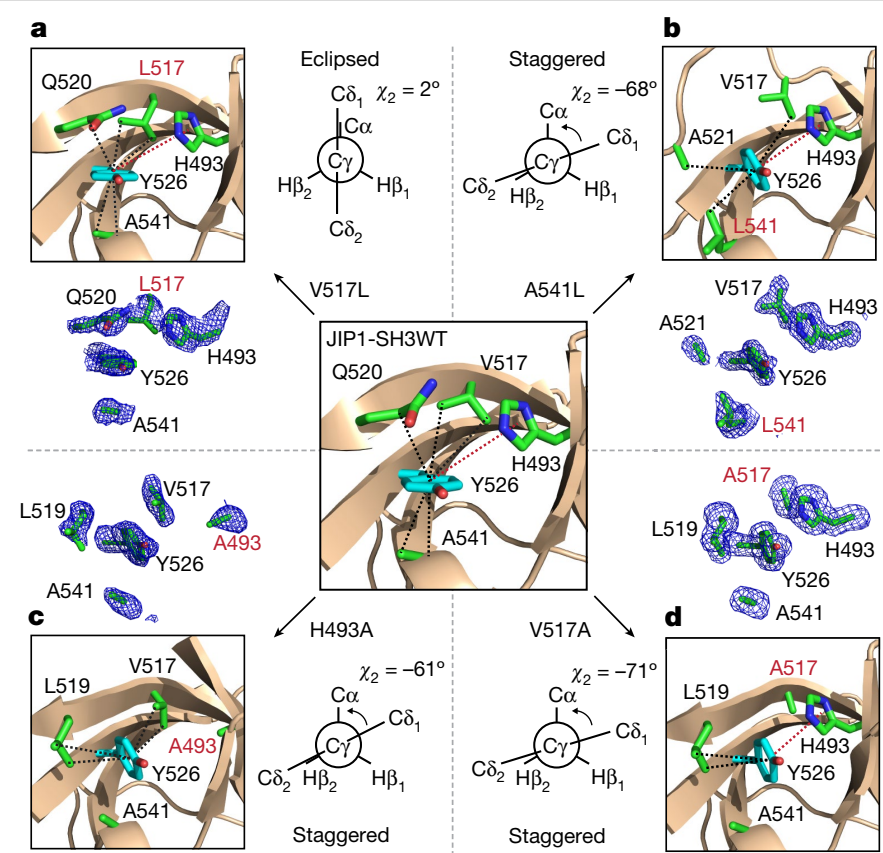

Fig. 2 | High-resolution crystal structures of JIP1-SH3 variants. a-d, Crystal structures showing the conformation of $\mathrm{Y} 526$, the corresponding unbiased electron density maps ( $\mathrm{Fo}-\mathrm{Fc}$ ) of $\mathrm{Y} 526$ and its surrounding residues, and the Newman projection along the $\mathrm{C} \beta-\mathrm{C} \gamma$ bond of $Y 526$ in the V517L (a), A541L (b), $\mathrm{H} 493 \mathrm{~A}(\mathbf{c})$ and V517A (d) variants of JIP1-SH3. Dashed lines indicate $\mathrm{CH}-\pi$ (black) and $\pi-\pi$ (red) interactions. Residue numbers in red indicate the site of mutation. The wild-type JIP1-SH3 structure is shown as a reference in the centre.

introducing single point mutations. The PCA suggests that a staggered conformation in group 2 is favoured over an eclipsed conformation in group 1 when residues with larger side chains are found in position 541 , when isoleucine or leucine are occupying position 517 and when smaller residues are found in position 493 (Fig. 1h, i). Accordingly, we designed four different mutants of JIP1-SH3 (H493A, V517A, V517L and $\mathrm{A} 541 \mathrm{~L}$ ) and obtained their high-resolution structures by X-ray crystallography (Extended Data Tables 1,2). Of note, three mutants (H493A, V517A and A541L) induce a staggered conformation of Y526, with $\chi_{2}$ dihedral angles ranging from $-41^{\circ}$ to $-75^{\circ}$, whereas $V 517 \mathrm{~L}$ shows an eclipsed conformation of Y526 and an almost identical structure to the wild-type protein (Fig. 2, Extended Data Fig. 6a). The high resolution of these structures, ranging from 1.4 to $1.9 \AA$, allows unambiguous determination of the conformation of Y526 and the surrounding residues, as demonstrated by their unbiased electron density maps (Fig. 2).

\section{Structural details of breathing motions}

The two variants H493A and V517A show almost identical crystal structures (Extended Data Fig. 6b) and an equivalent stabilization mechanism of the aromatic ring of Y526. Whereas the wild-type structure exhibits a classic $\beta$-bulge at residue 518 (ref. ${ }^{37}$ ), the transition from the eclipsed to the staggered conformation induces a local inversion (in-out) at residues 518-520, which leads to the formation of a canonical $\beta$-strand, as observed in the structures of the $\mathrm{H} 493 \mathrm{~A}$ and V517A variants (Fig. 3a-c). This transition allows the side chain of L519 to rearrange and form $\mathrm{CH}-\pi$ interactions with the ring of Y526 (Fig. 2c, d); and, at the same time, large-scale movements of E518, Q520, E522 and Y524 are observed (Fig. 3a). We note that $\mathrm{SH} 3$ domains in both group 1 and group 2 of the PCA show classic $\beta$-bulges at position 518 (Extended Data Fig. 5d), which suggests that the presence of this structural motif is not determinant of the side chain conformation of the phenyl ring in position 526.

The A541L mutation also triggers a staggered conformation of Y526 and a rearrangement of the 517-522 region; however, the stabilization mechanism of the aromatic ring is different. A looping out of the $\beta$-strand between residues 517 and 522 is observed (Extended Data Fig. 6c-e), which allows the side chain of A521 to reorient and to stabilize the staggered conformation of the ring through $\mathrm{CH}-\pi$ interactions together with L541 and V517 (Fig. 2b). At the same time, the side chains of L519, Q520 and E522 and D523 undergo large-scale movements to accommodate the flipped ring within the pocket (Extended Data Fig. 6c).

Altogether, the different mutants show that the dynamics of the region encompassing residues 517-522 are key for the formation of the minor state. The experimental ${ }^{13} \mathrm{C}$ chemical shifts for residues in this region are characteristic of random coil conformations (Extended Data Fig. 1b) and, compared to other regions of secondary structure, the relaxation-derived order parameters $\left(S^{2}\right)$ are lower (Extended Data Fig. 2g) and the crystallographic $B$-factors are higher. This supports the idea of the 517-522 region being intrinsically dynamic, with a fluctuating hydrogen-bonding network that is prone to structural transitions.

Next, we sought to determine which of the two crystal structures (H493A/V517A-like or A541L-like) best capture the conformation of the wild-type minor state detected by NMR relaxation dispersion. The $\mathrm{H} 493 \mathrm{~A}$ and V517A variants show almost identical crystal structures and for a subset of residues, the chemical shifts of which are unaffected by the mutations, the resonances of the two variants fall on a straight line together with the resonances of the wild-type protein (Fig.3d,e). This suggests that they are in fast-intermediate exchange between two conformations represented by the crystal structures of the wild-type protein and of the H493A/V517A variants. In agreement with this, both variants show line broadening and chemical exchange contributions as detected by ${ }^{15} \mathrm{~N}$ and ${ }^{1} \mathrm{H}^{\mathrm{N}}$ relaxation dispersion (Extended Data Figs. 7a-c, 8a-c). Analysis of these data (Extended Data Figs. 7d, e, 8d, e) shows that the structural features of the minor state of the wild-type protein are captured by the H493A/V517A crystal structures, as shown by the excellent agreement between the $\Delta \delta_{\mathrm{CPMG}}$ values for the wild-type protein and the two variants (Extended Data Figs. 7f, g, 8f, g). In addition, the analysis yields exchange rates between the staggered (canonical $\beta$-strand) and the eclipsed (classic $\beta$-bulge) conformation of $k_{\mathrm{EX}}=2,830 \pm 70 \mathrm{~s}^{-1}(\mathrm{H} 493 \mathrm{~A})$ and $k_{\mathrm{EX}}=6,800 \pm 300 \mathrm{~s}^{-1}(\mathrm{~V} 517 \mathrm{~A})$, compared to $k_{\mathrm{EX}}=2,600 \pm 70 \mathrm{~s}^{-1}$ determined for the wild-type protein. Finally, the observable chemical shifts of the two variants (Fig. 3d, e), in conjunction with analysis of the relaxation dispersion data (Supplementary Discussion), show that the H493A mutation slightly stabilizes the minor state relatively to the major state, whereas the V517A mutation almost inverts the relative populations of the major and minor states (Fig. 3f). For completeness, we note that the A541L crystal structure is not representative of the minor state conformation (Extended Data Fig. 9), although it shares structural features that are necessary for fast ring flipping of Y526 (see below).

\section{Void volume enables ring flipping}

Aromatic ${ }^{1} \mathrm{H}-{ }^{13} \mathrm{C}$ heteronuclear single quantum coherence (HSQC) spectra show averaging of the NMR signals of the tyrosine $\varepsilon_{1} / \varepsilon_{2}$ nuclei of Y526 (Fig. 4a, Extended Data Fig. 10a, Supplementary Fig. 1), which shows that full $180^{\circ}$ ring flipping occurs. This poses the question of the timescale of the full ring flipping and its relation to the observed minor state. To answer this question, we acquired L-optimized TROSY-selected aromatic side chain ${ }^{13} \mathrm{C} \varepsilon$ CPMG (ref. ${ }^{38}$ ) and on-resonance $R_{1 \rho}$ (ref. ${ }^{39}$ ) relaxation dispersion data of Y526. These data are entirely explained by the exchange process between the major and minor state, with a negligible contribution from the 


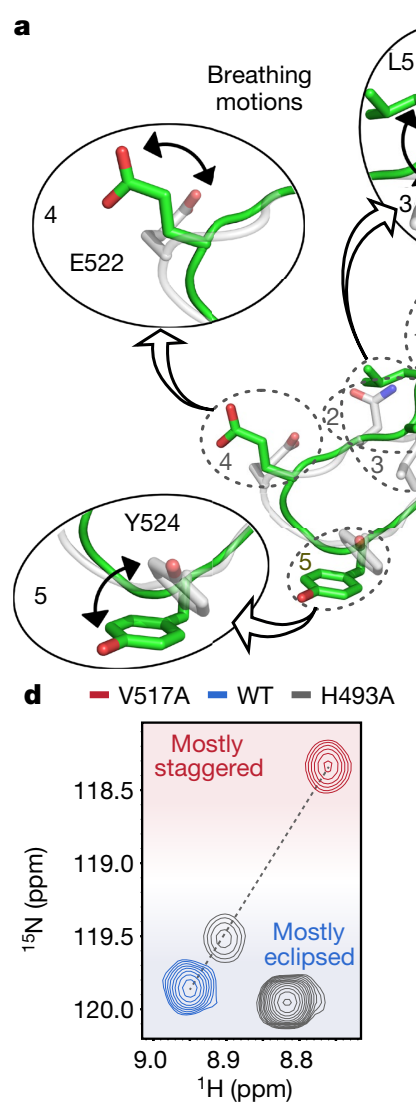

Fig. 3 | Crystal structures capture large-scale protein breathing motions. a, Structural changes associated with the transition from the eclipsed (green) to the staggered (grey) conformation of Y526. b, Illustration of the backbone conformation of the $\beta$-sheet formed between the 516-521 and 524-529 regions in the wild-type (WT) protein (left), and in the $\mathrm{H} 493 \mathrm{~A}$ (middle) and V517A (right) variants. Dashed lines indicate hydrogen bonds. $c$, Schematic representation of the conformation of the $\beta$-strand encompassing residues 516-521, showing the orientation of the carbonyl group ('out', carbonyl group surface exposed; 'in', carbonyl group pointing towards the $\beta$-strand encompassing residues

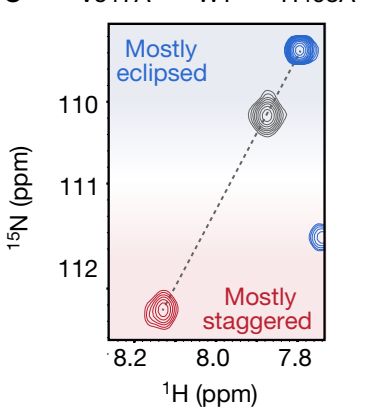

b WT
Classic $\beta$-bulge

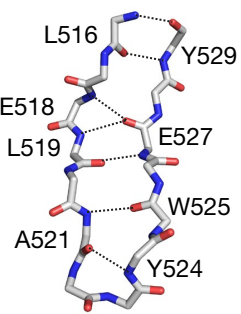

C Out $\underset{-}{\stackrel{\text { In }}{\rightarrow}}$
$\mathrm{H} 493 \mathrm{~A}$

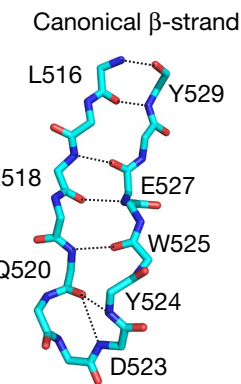

$\stackrel{\text { Out }}{\stackrel{\text { In }}{\rightarrow}}$
V517A

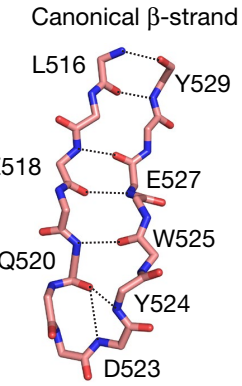

Out In

.

f

$W T$

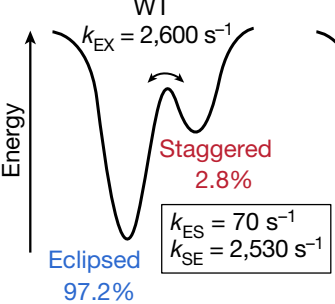

H493A

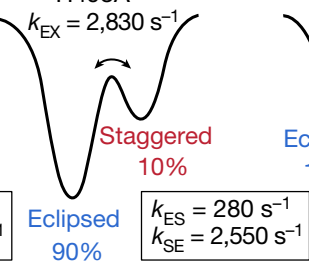

V517A

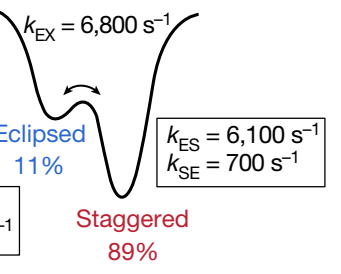

$89 \%$

524-529) in the wild-type protein (left) and in the H493A (middle) and V517A (right) variants. d, e, Two examples of linear correlations between the chemical shifts of wild-type JIP1-SH3 (blue spectrum) and the two variants H493A (grey spectrum) and V517A (red spectrum) as observed in ${ }^{1} \mathrm{H}-{ }^{15} \mathrm{~N} \mathrm{HSQC} \mathrm{spectra}$ acquired at $35^{\circ} \mathrm{C}(\mathbf{d}$, residue E522; e, residue D524).f, Energy landscapes illustrating the effect of single point mutations on the exchange rate constants and fractional populations of the major (eclipsed) and minor (staggered) conformations as determined by relaxation dispersion experiments acquired at $15^{\circ} \mathrm{C}$. full ring flipping event (Fig. 4b, c), demonstrating that ring flipping of Y526 is fast $\left(k_{\mathrm{EX}}>50,000 \mathrm{~s}^{-1}\right)$ (Supplementary Discussion). This observation agrees with a $1-\mu$ s molecular dynamics (MD) simulation that shows several $180^{\circ}$ ring flipping events of Y526 (Fig. 4d, Extended Data Fig. 10b, c).

During the structural transition between the major and the minor state (Supplementary Video 1), a void volume is created around the ring of Y526 that corresponds to a pocket expansion of $65 \AA^{3}$; this is mainly due to the structural reorganization of the side chain of Q520 (Fig. 4e,f). This cavity expansion is in agreement with previous studies that have reported activation volumes between 40 and $85 \AA^{3}$ for ring flipping events of aromatic residues in other proteins ${ }^{12,14,21,40,41}$. The expansion is followed by a compaction of the surrounding protein environment as the ring becomes stabilized by $\mathrm{CH}-\pi$ interactions from L519 (Fig. 4e, f).

Collectively, our results are consistent with a model in which fast protein breathing motions along the structural trajectory between the major and the minor state generate the necessary void volume for ring flipping to take place by lowering the energy of the transition state (Fig. 4e-g, Supplementary Video 2). Occasionally, the $\beta$-bulge to $\beta$-sheet transition is completed and the aromatic ring becomes trapped in a staggered conformation that is stabilized by $\mathrm{CH}-\pi$ interactions with L519-a process that gives rise to the observed relaxation dispersion. These events are rare and occur on a slow timescale (Figs. 3f, 4g), but they constitute an important tool for observing the trajectory of the protein breathing motions coupled to aromatic ring flipping. The initial generation of void volume around the ring is almost identical along the structural trajectory between the major state (wild type) and the A541L crystal structure (Extended Data Fig. 6f). Thus, all mutants-including A541L, which stabilizes Y526 in a staggered conformation by a different mechanism-share the same initial structural trajectory and report on identical breathing motions.

\section{Conclusions}

Our results provide structural insights into the protein breathing motions that are associated with aromatic ring flipping. We reveal how the dynamics of the region encompassing residues 517 to 522 are key for accommodating the ring flipping process of Y526. Notably, the transition from the eclipsed, major conformation to the staggered, minor conformation is associated with a structural change from a rare, classic $\beta$-bulge to a common, canonical $\beta$-strand conformation (Supplementary Video 1, Fig. 3a-c). Breathing motions along the structural trajectory between the major and the minor state generate the necessary void volume for fast ring flipping of Y526 to take place (Supplementary Video 2). Although a recent NMR study suggested extensive local unfolding as the source of cavity creation ${ }^{41}$, our study provides an alternative view by showing how a substantial void volume can be 

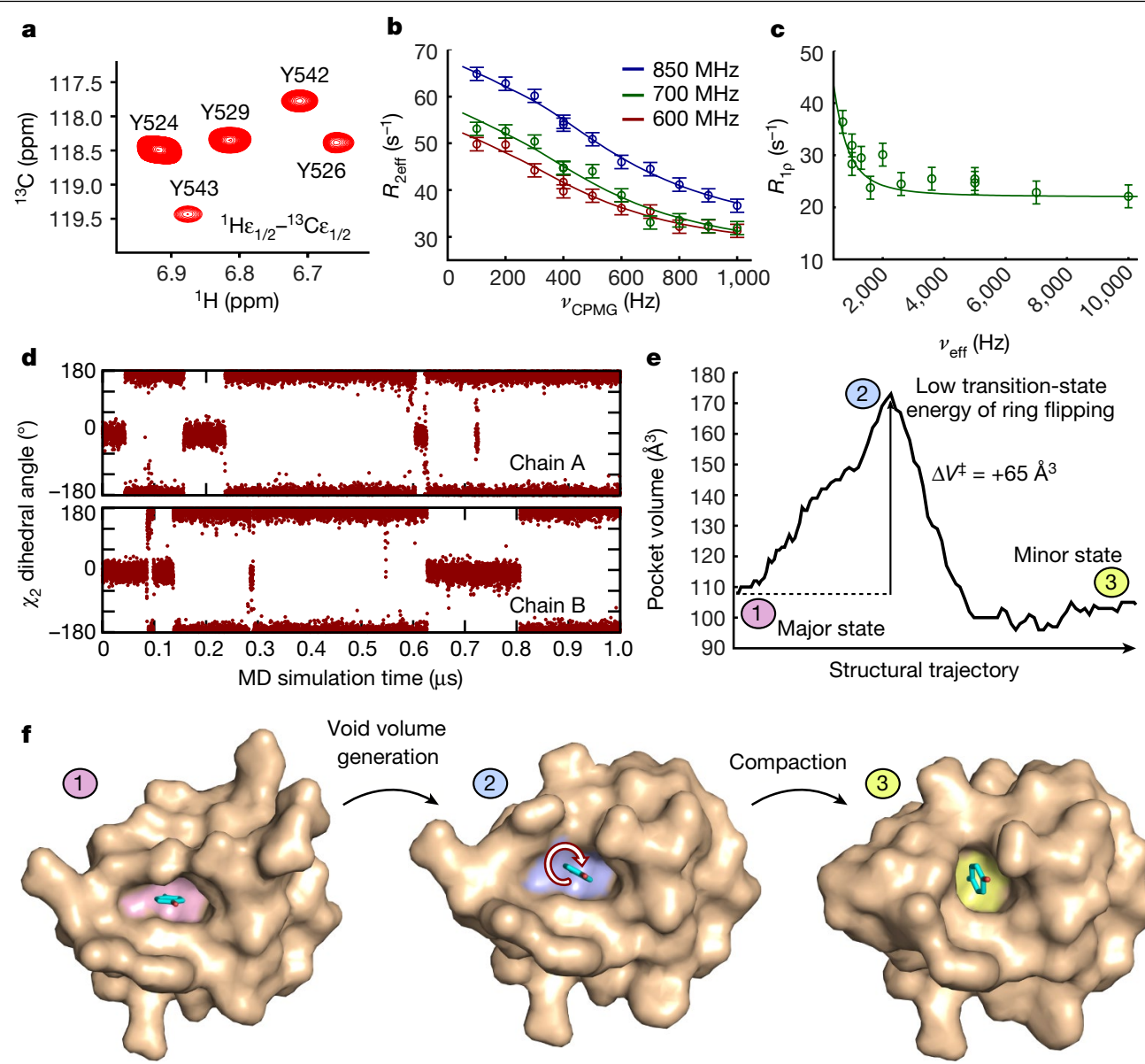

Void volume generation Compaction

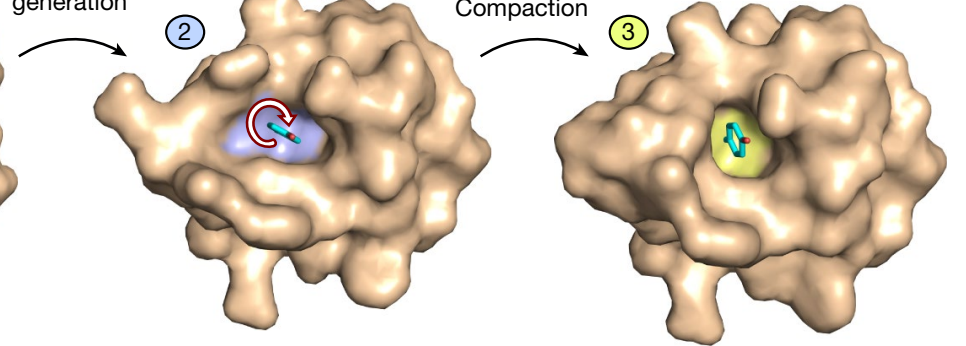

.

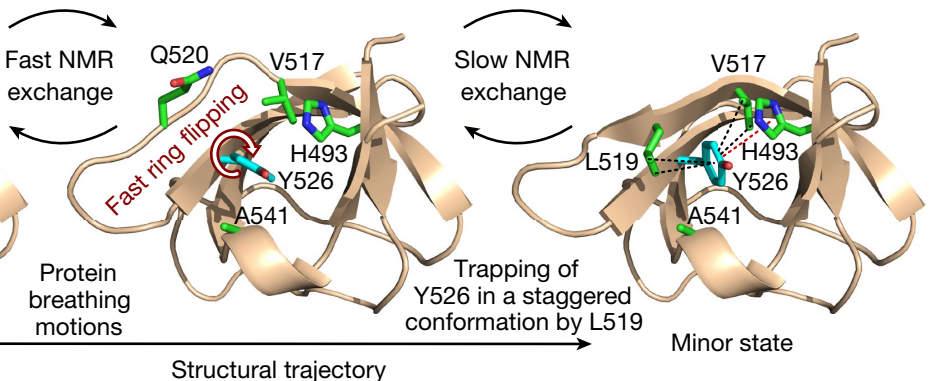

Fig. 4 Void volume creation enables fast aromatic ring flipping of Y526. a, ${ }^{1} \mathrm{H}^{-13} \mathrm{C}$ HSQC spectrum of JIP1-SH 3 at $15^{\circ} \mathrm{C}$ showing tyrosine epsilon correlations. b. ${ }^{13} \mathrm{C} \varepsilon \mathrm{CPMG}$ relaxation dispersion profiles of $\mathrm{Y} 526$ obtained at $15^{\circ} \mathrm{C}$ (red, $600 \mathrm{MHz}$; green, $700 \mathrm{MHz}$; blue, $850 \mathrm{MHz}$ ). The data were analysed simultaneously according to a two-site exchange model (full-drawn lines). Error bars represent one s.d. derived from Monte Carlo simulations of experimental uncertainty.c, ${ }^{13} \mathrm{C} \varepsilon$ on-resonance $R_{1 \rho}$ relaxation dispersion profile of $Y 526$ at $15^{\circ} \mathrm{C}$ and $700 \mathrm{MHz}$. The full-drawn line corresponds to the calculated $R_{1 \rho}$ profile from the exchange parameters $\left(k_{\mathrm{Ex}}, p_{\text {minor }}\right.$ and $\left.\Delta \delta_{\mathrm{CPMG}}\right)$ obtained from the analysis of the CPMG data in $\mathbf{b}$. Error bars represent one s.d. and were derived as in $\mathbf{b}$.d, The dihedral angle $X_{2}$ of $Y 526$ as a function of simulation time in a 1- $\mu \mathrm{s} \mathrm{MD} \mathrm{simulation} \mathrm{of} \mathrm{the} \mathrm{dimeric} \mathrm{JIP1-SH3.} \mathrm{e,} \mathrm{Volume} \mathrm{of} \mathrm{the}$ pocket of Y526 across the structural trajectory between the major and minor state.f, Surface representation of JIP1-SH3 in three different states corresponding to the major state, an intermediate state on the structural trajectory and the minor state. The Y526 pocket is highlighted in pink (major), blue (intermediate) and yellow (minor). The rearrangements along the structural trajectory between the major and the minor state generate a void volume around Y526, thereby lowering the transition-state energy of ring flipping. g, Illustration of the protein breathing motions along the structural trajectory from the major to the minor state. A void volume is created around Y526, which allows fast ring flipping to take place. The ring flipping is occasionally interrupted by trapping of Y526 in a staggered conformation through formation of $\mathrm{CH}-\pi$ interactions with $\mathrm{L} 519$ enabled by the $\beta$-bulge to $\beta$-sheet transition. generated through distinct structural rearrangements, while maintaining the overall protein fold.

More generally, our study shows how the local environment in the protein core can lead to a priori energetically unfavourable conformations of amino acid side chains, and how subtle changes in this environment can lead to major structural rearrangements to revert to preferred amino acid conformations. Our results therefore have implications for protein design and structure prediction, and for how novel biological functions can be acquired during the course of evolution; for example, by altering the delicate balance between hydrogen bonds and $\mathrm{CH}-\pi$ interactions. Finally, the combination of sensitive NMR methods to detect low-populated states, protein design using proteome-wide sequence analyses and high-resolution crystallography could be a strategy to further discover the structural details of sparsely populated protein states and their link to function. 


\section{Online content}

Any methods, additional references, Nature Research reporting summaries, source data, extended data, supplementary information, acknowledgements, peer review information; details of author contributions and competing interests; and statements of data and code availability are available at https://doi.org/10.1038/s41586-022-04417-6.

1. Campbell, I. D., Dobson, C. M. \& Williams, R. J. Proton magnetic resonance studies of the tyrosine residues of hen lysozyme-assignment and detection of conformational mobility. Proc. R. Soc. B. 189, 503-509 (1975).

2. Wüthrich, K. \& Wagner, G. NMR investigations of the dynamics of the aromatic amino acid residues in the basic pancreatic trypsin inhibitor. FEBS Lett. 50, 265-268 (1975).

3. Wagner, G., DeMarco, A. \& Wüthrich, K. Dynamics of the aromatic amino acid residues in the globular conformation of the basic pancreatic trypsin inhibitor (BPTI). I. ${ }^{1} \mathrm{H}$ NMR studies. Biophys. Struct. Mech. 2, 139-158 (1976).

4. Perutz, M. F. The role of aromatic rings as hydrogen-bond acceptors in molecular recognition. Philos. Trans. R. Soc. A 345, 105-112 (1993).

5. Brandl, M., Weiss, M. S., Jabs, A., Sühnel, J. \& Hilgenfeld, R. C-H...ா-interactions in proteins. J. Mol. Biol. 307, 357-377 (2001)

6. Steiner, T. \& Koellner, G. Hydrogen bonds with $\Pi$-acceptors in proteins: frequencies and role in stabilizing local 3D structures. J. Mol. Biol. 305, 535-557 (2001).

7. Burley, S. K. \& Petsko, G. A. Aromatic-aromatic interaction: a mechanism of protein structure stabilization. Science 229, 23-28 (1985).

8. McGaughey, G. B., Gagné, M. \& Rappé, A. K. ח-Stacking interactions. Alive and well in proteins. J. Biol. Chem. 273, 15458-15463 (1998).

9. Dougherty, D. A. Cation- $\pi$ interactions in chemistry and biology: a new view of benzene, Phe, Tyr, and Trp. Science 271, 163-168 (1996).

10. Ma, J. C. \& Dougherty, D. A. The cation-m interaction. Chem. Rev. 97, 1303-1324 (1997)

11. Wagner, G. \& Wüthrich, K. Dynamic model of globular protein conformations based on NMR studies in solution. Nature 275, 247-248 (1978)

12. Wagner, G. Activation volumes for the rotational motion of interior aromatic rings in globular proteins determined by high resolution ${ }^{1} \mathrm{H}$ NMR at variable pressure. FEBS Lett. 112, 280-284 (1980).

13. Skalicky, J. J., Mills, J. L., Sharma, S. \& Szyperski, T. Aromatic ring-flipping in supercooled water: implications for NMR-based structural biology of proteins. J. Am. Chem. Soc. 123, 388-397 (2001).

14. Hattori, M. et al. Infrequent cavity-forming fluctuations in HPr from Staphylococcus carnosus revealed by pressure- and temperature-dependent tyrosine ring flips. Protein Sci. 13, 3104-3114 (2004)

15. Rao, D. K. \& Bhuyan, A. K. Complexity of aromatic ring-flip motions in proteins: Y97 ring dynamics in cytochrome c observed by cross-relaxation suppressed exchange NMR spectroscopy. J. Biomol. NMR 39, 187-196 (2007)

16. Weininger, U., Respondek, M., Löw, C. \& Akke, M. Slow aromatic ring flips detected despite near-degenerate NMR frequencies of the exchanging nuclei. J. Phys. Chem. B 117, 9241-9247 (2013).

17. Weininger, U., Modig, K. \& Akke, M. Ring flips revisited: ${ }^{13} \mathrm{C}$ relaxation dispersion measurements of aromatic side chain dynamics and activation barriers in basic pancreatic trypsin inhibitor. Biochemistry 53, 4519-4525 (2014).

18. Yang, C.-J., Takeda, M., Terauchi, T., Jee, J. \& Kainosho, M. Differential large-amplitude breathing motions in the interface of FKBP12-drug complexes. Biochemistry 54, 6983-6995 (2015)

19. Kasinath, V., Fu, Y., Sharp, K. A. \& Wand, A. J. A sharp thermal transition of fast aromatic-ring dynamics in ubiquitin. Angew. Chem. Int. Ed. 54, 102-107 (2015).

20. Gauto, D. F. et al. Aromatic ring dynamics, thermal activation, and transient conformations of a $468 \mathrm{kDa}$ enzyme by specific ${ }^{1} \mathrm{H}{ }^{13} \mathrm{C}$ labeling and fast magic-angle spinning NMR. J. Am. Chem. Soc. 141, 11183-11195 (2019).

21. Dreydoppel, M., Raum, H. N. \& Weininger, U. Slow ring flips in aromatic cluster of GB1 studied by aromatic ${ }^{13} \mathrm{C}$ relaxation dispersion methods. J. Biomol. NMR 74, 183-191 2020).
22. Loria, J. P., Rance, M. \& Palmer, A. G. A relaxation-compensated Carr-Purcell-MeiboomGill sequence for characterizing chemical exchange by NMR spectroscopy. J. Am. Chem. Soc. 121, 2331-2332 (1999).

23. Ishima, R. \& Torchia, D. A. Extending the range of amide proton relaxation dispersion experiments in proteins using a constant-time relaxation-compensated CPMG approach. J. Biomol. NMR 25, 243-248 (2003).

24. Hansen, D. F., Vallurupalli, P. \& Kay, L. E. An improved ${ }^{15} \mathrm{~N}$ relaxation dispersion experiment for the measurement of millisecond time-scale dynamics in proteins. J. Phys. Chem. B 112, 5898-5904 (2008)

25. Yuwen, T. \& Kay, L. E. Revisiting ${ }^{1} H^{N}$ CPMG relaxation dispersion experiments: a simple modification can eliminate large artifacts. J. Biomol. NMR 73, 641-650 (2019).

26. Sugase, K., Dyson, H. J. \& Wright, P. E. Mechanism of coupled folding and binding of an intrinsically disordered protein. Nature 447, 1021-1025 (2007)

27. Baldwin, A. J. \& Kay, L. E. NMR spectroscopy brings invisible protein states into focus. Nat. Chem. Biol. 5, 808-814 (2009).

28. Bouvignies, G. et al. Solution structure of a minor and transiently formed state of a T4 lysozyme mutant. Nature 477, 111-114 (2011)

29. Neudecker, P. et al. Structure of an intermediate state in protein folding and aggregation. Science 336, 362-366 (2012).

30. Alderson, T. R. \& Kay, L. E. Unveiling invisible protein states with NMR spectroscopy. Curr. Opin. Struct. Biol. 60, 39-49 (2020).

31. Kristensen, $\mathrm{O}$. et al. A unique set of $\mathrm{SH} 3-\mathrm{SH} 3$ interactions controls IB1 homodimerization. EMBO J. 25, 785-797 (2006).

32. Dunbrack, R. L. \& Karplus, M. Conformational analysis of the backbone-dependent rotamer preferences of protein sidechains. Nat. Struct. Biol. 1, 334-340 (1994).

33. Teyra, J. et al. Comprehensive analysis of the human $\mathrm{SH} 3$ domain family reveals a wide variety of non-canonical specificities. Structure 25, 1598-1610 (2017).

34. Gehmlich, K. et al. Paxillin and ponsin interact in nascent costameres of muscle cells. J. Mol. Biol. 369, 665-682 (2007).

35. Hanawa-Suetsugu, K. et al. Structural basis for mutual relief of the Rac guanine nucleotide exchange factor DOCK2 and its partner ELMO1 from their autoinhibited forms. Proc. Natl Acad. Sci. USA 109, 3305-3310 (2012).

36. Borchert, T. V., Mathieu, M., Zeelen, J. P., Courtneidge, S. A. \& Wierenga, R. K. The crystal structure of human CskSH3: structural diversity near the RT-Src and n-Src loop. FEBS Lett. 341, 79-85 (1994).

37. Chan, A. W. E., Hutchinson, E. G., Harris, D. \& Thornton, J. M. Identification, classification, and analysis of $\beta$-bulges in proteins. Protein Sci. 2, 1574-1590 (1993).

38. Weininger, U., Respondek, M. \& Akke, M. Conformational exchange of aromatic side chains characterized by L-optimized TROSY-selected ${ }^{13} \mathrm{C}$ CPMG relaxation dispersion. J. Biomol. NMR 54, 9-14 (2012).

39. Weininger, U., Brath, U., Modig, K., Teilum, K. \& Akke, M. Off-resonance rotating-frame relaxation dispersion experiment for ${ }^{13} \mathrm{C}$ in aromatic side chains using L-optimized TROSY-selection. J. Biomol. NMR 59, 23-29 (2014)

40. Li, H., Yamada, H. \& Akasaka, K. Effect of pressure on the tertiary structure and dynamics of folded basic pancreatic trypsin inhibitor. Biophys. J. 77, 2801-2812 (1999).

41. Dreydoppel, M., Dorn, B., Modig, K., Akke, M. \& Weininger, U. Transition-state compressibility and activation volume of transient protein conformational fluctuations. JACS Au 1, 833-842 (2021).

Publisher's note Springer Nature remains neutral with regard to jurisdictional claims in published maps and institutional affiliations.

Open Access This article is licensed under a Creative Commons Attribution 4.0 International License, which permits use, sharing, adaptation, distribution and reproduction in any medium or format, as long as you give appropriate credit to the original author(s) and the source, provide a link to the Creative Commons license and indicate if changes were made. The images or other third party material in this article are included in the article's Creative Commons license, unless indicated otherwise in a credit line the material. If material is not included in the article's Creative Commons license and your intended use is not permitted by statutory regulation or exceeds the permitted use, you will need to obtain permission directly from the copyright holder. To view a copy of this license visit http://creativecommons.org/licenses/by/4.0/.

(c) The Author(s) 2022 


\section{Methods}

\section{Expression and purification of JIP1-SH3, POSH-SH3-1 and POSH-SH3-4}

JIP1-SH3, corresponding to residues 490-549 of human JIP1 (Uniprot Q9UQF2), was subcloned into a pET28a vector, and two of the four SH3 domains of the E3 ubiquitin-protein ligase SH3RF1 (Uniprot Q7Z6J0; also known as 'plenty of SH3 domains' (POSH)), SH3-1 (135-194) and SH3-4 (829-888), were subcloned into a pESPRIT vector ${ }^{42}$. The constructs therefore contained an $\mathrm{N}$-terminal hexahistidine tag followed by a tobacco etch virus (TEV) cleavage site. The final proteins after protease cleavage contain N-terminal GRR (POSH) or GHM extensions (JIP1).

To obtain unlabelled proteins, Escherichia coli BL21(DE3) cells transformed with one of the constructs were grown in lysogeny broth (LB) medium at $37^{\circ} \mathrm{C}$ until the optical density at $600 \mathrm{~nm}$ reached 0.7 . Protein expression was induced by the addition of isopropyl $\beta$-D1-thiogalactopyranoside (IPTG) to a final concentration of $1 \mathrm{mM}$. The cultures were grown for an additional $15 \mathrm{~h}$ at $20^{\circ} \mathrm{C}$ ( $\left.\mathrm{POSH}-\mathrm{SH} 3 \mathrm{~s}\right)$ or $4 \mathrm{~h}$ at $37^{\circ} \mathrm{C}$ (JIP1-SH3). The cells were collected by centrifugation and frozen at $-20^{\circ} \mathrm{C}$ or $-80{ }^{\circ} \mathrm{C}$. Isotopically ${ }^{15} \mathrm{~N} /{ }^{13} \mathrm{C}$ - and ${ }^{15} \mathrm{~N}$-labelled proteins were produced by growing transformed $E$. coli $\mathrm{BL} 21$ (DE3) cells in M9 minimal medium containing $1 \mathrm{~g} \mathrm{l}^{-1}$ of ${ }^{15} \mathrm{~N}-\mathrm{NH}_{4} \mathrm{Cl}$ and $2 \mathrm{~g} \mathrm{l}^{-1}$ of ${ }^{13} \mathrm{C}_{6}$-D-glucose or ${ }^{12} \mathrm{C}_{6}$ - D-glucose. To obtain ${ }^{15} \mathrm{~N}$-labelled protein with tyrosine residues site-selectively labelled at the $\varepsilon$ position with ${ }^{13} \mathrm{C}$, transformed $E$. coli BL21(DE3) cells were grown in M9 minimal medium containing $1 \mathrm{~g} \mathrm{l}^{-1}$ of ${ }^{15} \mathrm{~N}-\mathrm{NH}_{4} \mathrm{Cl}, 2 \mathrm{~g} \mathrm{l}^{-1}$ of $\mathrm{NaH}^{13} \mathrm{CO}_{3}$ and $2 \mathrm{~g} \mathrm{l}^{-1}$ of $\left[2-{ }^{13} \mathrm{C}\right]$-glycerol ${ }^{43}$.

All SH3 domains were purified by Ni affinity chromatography followed by size-exclusion chromatography. Cell lysis was carried out by sonication using purification buffer (POSH: $50 \mathrm{mM}$ Tris $\mathrm{pH}$ 7.0/8.0, $500 \mathrm{mM} \mathrm{NaCl}, 1 \mathrm{mM} \beta$-mercaptoethanol; JIP1: 50 mM HEPES pH 7.0, $150 \mathrm{mM} \mathrm{NaCl}$ ) supplemented with protease inhibitor tablets (Roche). The washing buffer used for Ni affinity chromatography was the same as the purification buffer with the addition of $20 \mathrm{mM}$ imidazole. The elution buffer was the same as the purification buffer with the addition of $500 \mathrm{mM}$ (POSH) or $300 \mathrm{mM}$ (JIP1) imidazole. Nickel affinity chromatography was followed by cleavage by the TEV protease, a second $\mathrm{Ni}$ affinity column and size-exclusion chromatography on a Superdex 75 (GE Healthcare). This column was equilibrated with $50 \mathrm{mM} \mathrm{HEPES} \mathrm{pH}$ 8.0, $500 \mathrm{mM} \mathrm{NaCl}, 2 \mathrm{mM}$ DTT for POSH-SH3-1, 50 mM HEPES pH 7.0, $500 \mathrm{mM} \mathrm{NaCl}, 2 \mathrm{mM}$ DTT for POSH-SH3-4 and $50 \mathrm{mM}$ HEPES pH 7.0, $150 \mathrm{mM} \mathrm{NaCl}$ for JIP1-SH3.

\section{Expression and purification of JIP1-SH3 variants}

Expression and purification of the JIP1-SH3 variants (Y526A, V517A, V517L, A541L and H493A) were performed following the same protocol as for JIP1-SH3, except that the cultures were grown for $15 \mathrm{~h}$ after induction at $20^{\circ} \mathrm{C}$ (instead of $4 \mathrm{~h}$ at $37^{\circ} \mathrm{C}$ ).

\section{Thermal stability measurements of JIP1-SH3}

The stability of JIP1-SH3 was measured by differential scanning fluorimetry using a Prometheus NT.48 (Nanotemper) instrument with the emission wavelengths set to 330 and $350 \mathrm{~nm}$ and an excitation power of $10 \%$. The melting curve for wild-type JIP1-SH3 was measured at a protein concentration of $4 \mathrm{mg} \mathrm{ml}^{-1}$ in $50 \mathrm{mMHEPES}, 150 \mathrm{mM} \mathrm{NaCl}$ at $\mathrm{pH} 7.0$ by using Prometheus Standard Capillaries (PR-C002). The temperature scan rate was fixed at $1^{\circ} \mathrm{C}$ per min from $20^{\circ} \mathrm{C}$ to $95^{\circ} \mathrm{C}$. The melting temperature $\left(T_{\mathrm{m}}\right)$ was calculated from the peak of the first derivative of the intrinsic protein fluorescence intensity ratio at $350 \mathrm{~nm}$ and $330 \mathrm{~nm}$ throughout the duration of the temperature ramp.

\section{NMR spectral assignment of JIP1-SH3 and its variants}

The NMR assignment experiments were acquired in $50 \mathrm{mM}$ HEPES, $150 \mathrm{mM} \mathrm{NaCl}, \mathrm{pH} 7.0$ at a protein concentration of $0.94 \mathrm{mM}$ (JIP1-SH3), 1.06 mM (JIP1-SH3(Y526A)), $1.10 \mathrm{mM}$ (JIP1-SH3(A541L)), $2 \mathrm{mM}$ (JIP1-SH3(V517A)) and $0.90 \mathrm{mM}$ (JIP1-SH3(H493A)). The NMR spectral assignments of JIP1-SH3 were performed at $25^{\circ} \mathrm{C}$ using a set of BEST-TROSY triple resonance experiments (HNCO, intra-residue HNCACO, HNCOCA, intra-residue HNCA, HNCOCACB and intra-residue $\mathrm{HNCACB}$ ) acquired at a ${ }^{1} \mathrm{H}$ frequency of $600 \mathrm{MHz}$ (Bruker, operated with TopSpin v.3.5) ${ }^{44}$. The NMR spectral assignments of JIP1-SH3(Y526A) were obtained at $25^{\circ} \mathrm{C}$ at a ${ }^{1} \mathrm{H}$ frequency of $700 \mathrm{MHz}$ (Bruker) using BEST-TROSY HNCO, HNCOCACB and intra-residue HNCACB experiments. The NMR spectral assignments of JIP1-SH3(A541L) (at $25^{\circ} \mathrm{C}$ ), JIP1-SH3(V517A) (at $35^{\circ} \mathrm{C}$ ) and JIP1-SH3(H493A) (at $35^{\circ} \mathrm{C}$ ) were obtained at a ${ }^{1} \mathrm{H}$ frequency of $700 \mathrm{MHz}$ (Bruker) using a BEST-TROSY HNCACB experiment. The spectra were manually peak-picked using NMRFAM-Sparky ${ }^{45}$ and sequential connectivities were identified manually or by using the assignment program MARS ${ }^{46}$. Secondary structure propensities were calculated using SSP on the basis of the experimental $\mathrm{C} \alpha$ and $\mathrm{C} \beta$ chemical shifts ${ }^{47}$.

\section{${ }^{15} \mathrm{~N}$ relaxation measurements of JIP1-SH3}

Measurements of ${ }^{15} \mathrm{~N}$ relaxation rates $\left(R_{1}, R_{2}\right.$ and heteronuclear NOEs) of JIP1-SH3 were obtained using standard HSQC-type pulse sequences ${ }^{48}$ at ${ }^{1} \mathrm{H}$ frequency of $600 \mathrm{MHz}$ (Agilent, operated with VnmrJ v.3.1). The relaxation rates were measured at four different temperatures: 15, 25, 35 and $45^{\circ} \mathrm{C}$. The magnetization decay was sampled at $(0,100,200,400$, $600,800,1,100,1,500$ and 1,900) milliseconds ( $\mathrm{ms}$ ) for longitudinal and at $(10,30,50,70,90,130,170,210$ and 250$)$ ms for transverse relaxation. Technical replicates of one or two of these delays were acquired to estimate the uncertainty on the relaxation rates using a Monte Carlo approach. Details of the Lipari-Szabo model free analysis can be found in the Supplementary Discussion.

${ }^{15} \mathrm{~N}$ and ${ }^{1} \mathrm{H}^{\mathrm{N}} \mathrm{CPMG}$ relaxation dispersion of JIP1-SH3 and its variants All ${ }^{15} \mathrm{~N}$ CPMG relaxation dispersion experiments ${ }^{24}$ were carried out at $15^{\circ} \mathrm{C}$ using a constant-time relaxation delay of $32 \mathrm{~ms}$ with CPMG frequencies $\left(v_{\text {CPMG }}\right)$ ranging from 31.25 to $1,000 \mathrm{~Hz}$ and ${ }^{1}{ }^{1} \mathrm{H}$ decoupling field of $11 \mathrm{kHz}$. The ${ }^{1} \mathrm{H}^{\mathrm{N}}$ relaxation dispersion experiments were carried out at $15^{\circ} \mathrm{C}$ using the published pulse sequence ${ }^{25}$ with a constant-time relaxation delay of $20 \mathrm{~ms}$ and CPMG frequencies ranging from 50 to $2,000 \mathrm{~Hz}$. Uncertainties on peak intensities extracted from the relaxation dispersion experiments were estimated using the pooled s.d. calculated from repeat measurements (technical replicates of one to three $v_{\mathrm{CPMG}}$ values), each pool being the set of repeat points per $v_{\mathrm{CPMG}}$ and per peak. Uncertainties on $R_{\text {2eff }}$ values were propagated from the peak intensity uncertainty using a Monte Carlo approach. The following relaxation dispersion experiments were acquired:JIP1-SH3: ${ }^{15} \mathrm{~N}$ (600 MHz, Agilent), ${ }^{15} \mathrm{~N}\left(850 \mathrm{MHz}\right.$, Bruker), ${ }^{1} \mathrm{H}^{\mathrm{N}}(600 \mathrm{MHz}$, Bruker $),{ }^{1} \mathrm{H}^{\mathrm{N}}$ (950 MHz, Bruker);JIP1-SH3(H493A) and JIP1-SH3(V517A): ${ }^{15} \mathrm{~N}(600 \mathrm{MHz}$, Bruker), ${ }^{15} \mathrm{~N}$ (950 MHz, Bruker), ${ }^{1} \mathrm{H}^{\mathrm{N}}$ (600 MHz, Bruker), ${ }^{1} \mathrm{H}^{\mathrm{N}}$ (950 $\mathrm{MHz}$, Bruker); JIP1-SH3(Y526A): ${ }^{15} \mathrm{~N}\left(700 \mathrm{MHz}\right.$, Bruker) and ${ }^{1} \mathrm{H}^{\mathrm{N}}$ (600 MHz, Bruker);JIP1-SH3(A541L): ${ }^{15} \mathrm{~N}$ (700 MHz, Bruker). All relaxation dispersion data were analysed using the program ChemEx (https:// github.com/gbouvignies/ChemEx ${ }^{49}$ as described in the Supplementary Discussion.

Tyrosine assignments and ${ }^{13} \mathrm{C} \mathrm{CPMG} \mathrm{relaxation} \mathrm{dispersion} \mathrm{of} \mathrm{Y526}$ The ${ }^{13} \mathrm{C} \varepsilon^{-1} \mathrm{H} \varepsilon$ tyrosine resonances were assigned at $45^{\circ} \mathrm{C}$ by acquiring a two-dimensional (2D) plane of a BEST-TROSY intra-residue HNCACB experiment $\mathrm{t}^{44}$, an aromatic BEST constant-time ${ }^{1} \mathrm{H}-{ }^{13} \mathrm{C}$ HSQC experiment ${ }^{50}$ and a $(\mathrm{H} \beta) \mathrm{C} \beta(\mathrm{C} \gamma \mathrm{C} \delta \mathrm{C} \varepsilon) \mathrm{H} \varepsilon$ experiment ${ }^{51}$ linking the $\mathrm{C} \beta$ chemical shifts directly to the $\mathrm{H} \varepsilon$ chemical shifts. The spectra were manually peak-picked using NMRFAM-Sparky ${ }^{45}$ and ${ }^{13} \mathrm{C} \varepsilon^{-1} \mathrm{H} \varepsilon$ tyrosine resonances were assigned manually (Supplementary Fig. 1). The acquisition of aromatic BEST constant-time ${ }^{1} \mathrm{H}-{ }^{13} \mathrm{C}$ HSQC experiments at different temperatures (between 5 and $45^{\circ} \mathrm{C}$ ) enabled the final assignment at $15^{\circ} \mathrm{C}$.

Aromatic L-optimized TROSY-selected ${ }^{13} \mathrm{CCPMG}^{38}$ and $R_{10}{ }^{39}$ relaxation dispersion experiments were carried out at $15^{\circ} \mathrm{C}$ on a $1 \mathrm{mM}$ uniformly ${ }^{15} \mathrm{~N}$ and site-selective ${ }^{13} \mathrm{C}$-labelled JIP1-SH3 sample in $50 \mathrm{mM}$ 
HEPES, $150 \mathrm{mM} \mathrm{NaCl}$ at $\mathrm{pH}$ 7.0. CPMG relaxation dispersion experiments were carried out at magnetic field strengths of $600 \mathrm{MHz}$, $700 \mathrm{MHz}$ and $850 \mathrm{MHz}$ (Bruker) using a constant-time relaxation delay of $20 \mathrm{~ms}$ with CPMG frequencies ranging from 100 to $1,000 \mathrm{~Hz}$. The $R_{1 \mathrm{p}}$ relaxation dispersion experiment was recorded on-resonance with Y526 at $700 \mathrm{MHz}$ (Bruker) using $B_{1}$ field strengths ranging from 700 to $10,000 \mathrm{~Hz}$ with a $20 \mathrm{~ms}$ relaxation delay. Error bars were derived from repeat measurements as described above for the ${ }^{15} \mathrm{~N}$ and ${ }^{1} \mathrm{H}^{\mathrm{N}}$ relaxation dispersion experiments. Analysis of the ${ }^{13} \mathrm{C}$ data was carried out using ChemEx or using available analytical expressions for $R_{1 \rho}$ relaxation in the presence of two-site exchange ${ }^{52}$ (Supplementary Discussion).

\section{Comparison of JIP1-SH3 to other human SH3 domains}

The sequences of 320 human SH3 domains were obtained ${ }^{33}$, aligned using Clustal Omega ${ }^{53}$ and categorized according to the identity of the amino acid at the position of Y526 in JIP1-SH3. A new alignment was performed using only the sequences that carry $Y$ or $F$ at this position, amounting to a total of 33 human $\mathrm{SH} 3$ domains. The sequences of the $\mathrm{SH} 3$ domains of the RIMS-binding proteins 1, 2 and 3 and the metastasis-associated in colon cancer protein 1 (MACC1) were not included in this alignment, as they contain longer insertions compared to the JIP1-SH3 sequence. For each SH3 domain, the amino acids corresponding to residues 493,517 and 541 of JIP1-SH3 were assigned a size score according to the number of heavy atoms in their side chains (A:1, C:2, D: 4, E: 5, F: 7, G: 0, H: 6, I: 4, K: 5, L:4, M: 4, N: 4, P:3, Q: 5, R: 7, $\mathrm{S}: 2, \mathrm{~T}: 3, \mathrm{~V}: 3, \mathrm{Y}: 8, \mathrm{~W}: 10)$. A PCA was carried out to reveal potential correlations between the sizes of the amino acids in position 493, 517 and 541 using the ClustVis webtool ${ }^{54}$.

\section{Crystallization of JIP1-SH3 and the variants Y526A, V517A, V517L, A541L and H493A}

JIP1-SH3 and its variants were concentrated to a final concentration of $20 \mathrm{mg} \mathrm{ml}^{-1}$ (JIP1-SH3, JIP1-SH3(V517A), JIP1-SH3(A541L) and JIP1-SH3(V517L)), $10 \mathrm{mg} \mathrm{ml}^{-1}$ (JIP1-SH3(Y526A)) and $4 \mathrm{mg} \mathrm{ml}^{-1}$ (JIP1-SH3(H493A)) after size-exclusion chromatography by using Amicon Ultra-4 3.0-kDa centrifugal filters (Merck). All crystals were obtained in 0.1 M HEPES pH 7.5, 1-5\% PEG 400 and 2-2.5 M ammonium sulfate at $20^{\circ} \mathrm{C}$ by the hanging-drop vapour diffusion method in 24-well plates (Hampton research) ${ }^{55}$. Drops of 2-3 $\mu$ l consisting of 1:1 or 2:1 parts of protein solution and reservoir solution were vapour-equilibrated against $500 \mu \mathrm{l}$ of reservoir solution. All crystals appeared after two days and were collected by transferring them to a mother liquor solution containing 20-30\% trehalose, frozen and kept in liquid nitrogen.

\section{Crystallization of POSH-SH3-1 and POSH-SH3-4}

Purified POSH-SH3-1 and POSH-SH3-4 were directly concentrated after size-exclusion chromatography to 3.4 and $5.0 \mathrm{mg} \mathrm{ml}^{-1}$, respectively, using Amicon Ultra-43.5-kDa centrifugal filters (Merck). Initial crystallization conditions were identified using the high-throughput crystallization platform (EMBL).

The initial condition identified for POSH-SH3-1 was $0.2 \mathrm{M} \mathrm{NaF}$, $20 \%$ PEG 3350 from the PEGs-I screen (Qiagen) at $4{ }^{\circ} \mathrm{C}$. Needles appeared after 3 to 7 days. Further optimization was done using the hanging-drop vapour diffusion method at $4{ }^{\circ} \mathrm{C}$ in 24 -well plates. Drops of $2 \mu \mathrm{l}$ consisting of equal parts protein solution at $2.5 \mathrm{mg} \mathrm{ml}^{-1}$ and reservoir solution (0.2 M NaF, 22\% PEG 3350) were vapour-equilibrated against $500 \mu \mathrm{l}$ of reservoir solution. Hexagonal crystals appeared after three days and were collected after five days by transferring them to a mother liquor solution containing $5 \%$ ethylene glycol as cryoprotectant, frozen and kept in liquid nitrogen.

The initial screen of POSH-SH3-4 identified two crystallization conditions: 0.1 M MES pH 6.5, 25\% PEG 3000 (condition 1) and 0.1 M MES pH 6.5, 25\% PEG 4000 (condition 2) from the PEGs-I screen (Qiagen) at $4{ }^{\circ} \mathrm{C}$. Diffraction-quality needles (condition $1,0.1 \mathrm{M} \mathrm{MES} \mathrm{pH} \mathrm{6.5,}$ 26\% PEG 3000) or three-dimensional crystals (condition 2, $0.1 \mathrm{M} \mathrm{MES}$ $\mathrm{pH}$ 6.5, 23\% PEG 4000) were obtained after four days using the same vapour-diffusion set-up as for POSH-SH3-1. These were collected after seven days with $10 \%$ ethylene glycol as cryoprotectant, frozen and kept in liquid nitrogen.

\section{Structure determination}

Crystal diffraction was performed at the ESRF beamlines ID30A, ID23-1, ID23-2 using the MXCube software ${ }^{56,57}$, at the automated beamline MASSIF- ${ }^{58}$ or at the Diamond beamlines I04 and I04-1, all equipped with Pilatus detectors (Dectris). Indexing and integration was performed using the XDS ${ }^{59}$, the autoProc ${ }^{60}$ or GrenADeS ${ }^{61}$ program suites. Data reduction for JIP1-SH3(H493A) was carried out with Pointless and Aimless $^{62,63}$. Molecular replacement of the wild-type JIP1-SH3 structure was carried out in Phaser $^{64}$ using the PDB code $2 \mathrm{FPE}$ (chains A-B) as a search model. The structures of JIP1-SH3 mutants were obtained by using our wild-type JIP1-SH3 structure as a search model. The initial solutions were improved through cycles of manual adjusting in $\operatorname{Coot}^{65}$ and refined by using Refmac $5^{66}$. Aimless, Phaser and Refmac were all used as programs of the CCP4 suite ${ }^{67}$.

The structure of POSH-SH3-4 was determined by molecular replacement using a homology model that was built on the basis of the $\mathrm{SH} 3$ domain structure of SORBS1 (PDB code: $2 \mathrm{LJ1}$, chain A), which has $45 \%$ sequence identity. The structure of POSH-SH3-1 was determined by molecular replacement using as a search model the $\mathrm{SH} 3$ domain of human tyrosine protein kinase C-Src (PDB code:2SRC). Crystallography applications were compiled and configured by SBGrid ${ }^{68}$.

\section{Structural trajectory and void volume calculations}

The structural trajectory between the major and minor conformation was generated with Chimera ${ }^{69}$ by morphing between the wild-type JIP1-SH3 structure (PDB 7NYK) and the structures of the two variants JIP1-SH3(H493A) (7NYL) and JIP1-SH3(V517A) (7NYM). To calculate changes in the volume of the Y526 pocket, protons were added to all structures of the trajectory and Y526 was replaced by glycine to allow calculation of the complete pocket volume by POVME 3.0 using a distance cut-off of $1.09 \AA$, corresponding to the van der Waals radius of a hydrogen atom ${ }^{70}$. A similar strategy was used to generate the structural trajectory between the wild-type JIP1-SH3 structure (PDB 7NYK) and the structure of the JIP1-SH3(A541L) variant (7NYO).

\section{MD simulations of JIP1-SH3}

MD simulations were carried out using ACEMD v.3.3.0 $0^{71}$ and the Charmm $36 \mathrm{~m}$ force field parameters ${ }^{72}$. Using VMD ${ }^{73}$, coordinates of the dimer from PDB 2FPE were inserted in the box of dimensions with a minimum distance of $2 \AA$ in each direction between each atom and any box side. The box was then filled with water molecules and an amount of $\mathrm{Na}^{+}$and $\mathrm{Cl}^{-}$corresponding to $[\mathrm{NaCl}]=0.1 \mathrm{M}$. Electrostatic interactions were evaluated using Particle-Mesh Ewald (PME) electrostatics with a cut-off distance of $9 \AA$. Van der Waals forces were calculated with a cut-off of $9 \AA$ and a switching function active from $7.5 \AA$ to smoothly reduce the potential to zero. An integration step of $2 \mathrm{fs}$ and holonomic constraints on all hydrogen-heavy atom bond terms were used. The energy of the system was minimized using conjugate-gradient minimization for 500 steps. Random velocities from a Maxwell distribution with $T=298.15 \mathrm{~K}$ were assigned to atoms. Then, the system was equilibrated first for 100 ps in the NVE ensemble and then for $1 \mathrm{~ns}$ in the NPT ensemble. In the latter case, temperature and pressure were controlled using the Langevin thermostat with a damping constant of $1 \mathrm{ps}^{-1}$ and Berendsen barostat with a relaxation time of $400 \mathrm{fs}$, respectively. Finally, a $1 \mu$ s trajectory was calculated in the NVT ensemble using the Langevin thermostat with a damping constant of $0.1 \mathrm{ps}^{-1}$. Trajectories were processed and analysed using the MDAnalysis Python package ${ }^{74}$. 


\section{Reporting summary}

Further information on research design is available in the Nature Research Reporting Summary linked to this paper.

\section{Data availability}

Protein structure data have been deposited in the PDB with accession codes: 7NYK (JIP1-SH3), 7NZB (JIP1-SH3(V517L)), 7NYO (JIP1-SH3(A541L)), 7NYL (JIP1-SH3(H493A)), 7NYM (JIP1-SH3(V517A)), 7NZC (POSH-SH3-1) and 7NZD (POSH-SH3-4). The ${ }^{1} \mathrm{H}^{13}{ }^{13}$ and ${ }^{15} \mathrm{~N}$ chemical shifts of JIP1-SH3 have been deposited in the Biological Magnetic Resonance Data Bank with accession codes: 50814 (JIP1-SH3), 50817 (JIP1-SH3(Y526A)), 50816 (JIP1-SH3(V517A)), 50818 (JIP1-SH3(H493A)) and 50815 (JIP1-SH3(A541L)). SH3 domain structures for molecular replacement were retrieved from the PDB (https://www.ebi.ac.uk/ pdbe/) with accession codes: 2FPE (JIP1), 2LJ1 (SORBS1) and 2SRC (tyrosine protein kinase C-Src). Structures for the proteome-wide SH3 sequence analysis were retrieved from the PDB with accession codes: 1CSK, 3A98, 2O9S, 5VEI and 4LNP.

42. Hart, D. J. \& Tarendeau, F. Combinatorial library approaches for improving soluble protein expression in Escherichia coli. Acta Crystallogr. D 62, 19-26 (2006).

43. LeMaster, D. M. \& Kushlan, D. M. Dynamical mapping of E. coli thioredoxin via ${ }^{13} \mathrm{C}$ NMR relaxation analysis. J. Am. Chem. Soc. 118, 9255-9264 (1996).

44. Solyom, Z. et al. BEST-TROSY experiments for time-efficient sequential resonance assignment of large disordered proteins. J. Biomol. NMR 55, 311-321 (2013).

45. Lee, W., Tonelli, M. \& Markley, J. L. NMRFAM-SPARKY: enhanced software for biomolecular NMR spectroscopy. Bioinformatics 31, 1325-1327 (2015)

46. Jung, Y.-S. \& Zweckstetter, M. Mars-robust automatic backbone assignment of proteins. J. Biomol. NMR 30, 11-23 (2004).

47. Marsh, J. A., Singh, V. K., Jia, Z. \& Forman-Kay, J. D. Sensitivity of secondary structure propensities to sequence differences between alpha- and gamma-synuclein: implications for fibrillation. Protein Sci. 15, 2795-2804 (2006).

48. Farrow, N. A. et al. Backbone dynamics of a free and phosphopeptide-complexed Src homology 2 domain studied by ${ }^{15} \mathrm{~N}$ NMR relaxation. Biochemistry 33, 5984-6003 (1994).

49. Vallurupalli, P., Bouvignies, G. \& Kay, L. E. Studying 'invisible' excited protein states in slow exchange with a major state conformation. J. Am. Chem. Soc. 134, 8148-8161 (2012).

50. Christou, N. E. \& Brutscher, B. BEST and SOFAST experiments for resonance assignment of histidine and tyrosine side chains in ${ }^{13} \mathrm{C} /{ }^{15} \mathrm{~N}$ labeled proteins. J. Biomol. NMR 72, 115-124 (2018).

51. Yamazaki, T., Forman-Kay, J. D. \& Kay, L. E. Two-dimensional NMR experiments for correlating ${ }^{13} \mathrm{C} \beta$ and ${ }^{1} \mathrm{H} \delta / \varepsilon$ chemical shifts of aromatic residues in ${ }^{13} \mathrm{C}$-labeled proteins via scalar couplings. J. Am. Chem. Soc. 115, 11054-11055 (1993).

52. Miloushev, V. Z. \& Palmer, A. G. $R_{10}$ relaxation for two-site chemical exchange: general approximations and some exact solutions. J. Magn. Reson. 177, 221-227 (2005).

53. Madeira, F. et al. The EMBL-EBI search and sequence analysis tools APIs in 2019. Nucleic Acids Res. 47, W636-W641 (2019).

54. Metsalu, T. \& Vilo, J. ClustVis: a web tool for visualizing clustering of multivariate data using principal component analysis and heatmap. Nucleic Acids Res. 43, W566-W570 (2015).

55. Dar, I., Bonny, C., Pedersen, J. T., Gajhede, M. \& Kristensen, O. Crystallization and preliminary crystallographic characterization of an $\mathrm{SH} 3$ domain from the IB1 scaffold protein. Acta Crystallogr. D 59, 2300-2302 (2003).

56. Gabadinho, J. et al. MxCuBE: a synchrotron beamline control environment customized for macromolecular crystallography experiments. J. Synchrotron Radiat. 17, 700-707 (2010)

57. Oscarsson, M. et al. MXCuBE2: the dawn of MXCuBE collaboration. J. Synchrotron Radiat 26, 393-405 (2019).

58. Svensson, O., Malbet-Monaco, S., Popov, A., Nurizzo, D. \& Bowler, M. W. Fully automatic characterization and data collection from crystals of biological macromolecules. Acta Crystallogr. D 71, 1757-1767 (2015).

59. Kabsch, W. XDS. Acta Crystallogr. D 66, 125-132 (2010).
60. Vonrhein, C. et al. Data processing and analysis with the autoPROC toolbox. Acta Crystallogr. D 67, 293-302 (2011).

61. Monaco, S. et al. Automatic processing of macromolecular crystallography X-ray diffraction data at the ESRF. J. Appl. Crystallogr. 46, 804-810 (2013).

62. Evans, P. R. An introduction to data reduction: space-group determination, scaling and intensity statistics. Acta Crystallogr. D 67, 282-292 (2011).

63. Evans, P. R. \& Murshudov, G. N. How good are my data and what is the resolution? Acta Crystallogr. D 69, 1204-1214 (2013)

64. McCoy, A. J. et al. Phaser crystallographic software. J. Appl. Crystallogr. 40, 658-674 (2007).

65. Emsley, P., Lohkamp, B., Scott, W. G. \& Cowtan, K. Features and development of Coot. Acta Crystallogr. D 66, 486-501 (2010).

66. Murshudov, G. N. et al. REFMAC5 for the refinement of macromolecular crystal structures. Acta Crystallogr. D 67, 355-367 (2011).

67. Winn, M. D. et al. Overview of the CCP4 suite and current developments. Acta Crystallogr. D 67, 235-242 (2011)

68. Morin, A. et al. Collaboration gets the most out of software. eLife 2, e01456 (2013).

69. Pettersen, E. F. et al. UCSF Chimera-a visualization system for exploratory research and analysis. J. Comput. Chem. 25, 1605-1612 (2004)

70. Wagner, J. R. et al. POVME 3.0: software for mapping binding pocket flexibility. J. Chem. Theory Comput. 13, 4584-4592 (2017).

71. Harvey, M. J., Giupponi, G. \& Fabritiis, G. D. ACEMD: accelerating biomolecular dynamics in the microsecond time scale. J. Chem. Theory Comput. 5, 1632-1639 (2009).

72. Huang, J. et al. CHARMM $6 \mathrm{~m}$ : an improved force field for folded and intrinsically disordered proteins. Nat. Methods 14, 71-73 (2017).

73. Humphrey, W., Dalke, A. \& Schulten, K. VMD: visual molecular dynamics. J. Mol. Graph. 14, $27-28$ (1996)

74. Michaud-Agrawal, N., Denning, E. J., Woolf, T. B. \& Beckstein, O. MDAnalysis: a toolkit for the analysis of molecular dynamics simulations. J. Comput. Chem. 32, 2319-2327 (2011).

75. Dosset, P., Hus, J. C., Blackledge, M. \& Marion, D. Efficient analysis of macromolecular rotational diffusion from heteronuclear relaxation data. J. Biomol. NMR 16, 23-28 (2000).

76. Krissinel, E. \& Henrick, K. Inference of macromolecular assemblies from crystalline state. J. Mol. Biol. 372, 774-797 (2007)

Acknowledgements We thank W. Adamski, S. Milles and T. Herrmann for discussions. We acknowledge the platforms of the Grenoble Instruct European Research Infrastructure Consortium (Integrated Structural Biology Grenoble; UAR 3518 CNRS-CEA-UGA-EMBL) within the Grenoble Partnership for Structural Biology. Platform access was supported by French Infrastructure for Integrated Structural Biology (ANR-10-INBS-05-02) and the Grenoble Alliance for Integrated Structural and Cell Biology, a project of the University Grenoble Alpes graduate school (Ecoles Universitaires de Recherche) CBH-EUR-GS (ANR-17-EURE-0003). The Institut de Biologie Structurale acknowledges integration into the Interdisciplinary Research Institute of Grenoble. The authors thank the Diamond Light Source and the European Synchrotron Radiation Facility for beamtime access and technical support. This work was funded by the Fondation pour la Recherche Medicale through a postdoctoral fellowship to L.M.P. (contract SPF201909009258) and by a PhD fellowship to J.K. from the IDPbyNMR Marie Curie action of the European commission (contract no. 264257). Funding is also acknowledged from the Agence National de la Recherche (ANR) through ANR T-ERC MAPKassembly (to M.R.J.), ANR ScaffoldDisorder (to M.R.J. and A.P.) and ANR JCJC RC18114CC NovoTargetParasite (to A.P.)

Author contributions L.M.P., M.R.J. and A.P. conceived the study. L.M.P., L.M.B., D.M. and J.K. made samples. L.M.B. and A.P. solved the structures of the POSH SH3 domains. L.M.P., F.S.I. and A.P. solved the structures of JIP1-SH3 and its variants. L.M.P., J.K. and M.R.J. designed and performed all NMR experiments. L.M.P., G.B. and M.R.J. analysed and interpreted the NMR data. M.B. and N.S. performed and analysed the MD simulation. M.R.J. and A.P. wrote the paper with input from all authors.

Competing interests The authors declare no competing interests.

\section{Additional information}

Supplementary information The online version contains supplementary material available at https://doi.org/10.1038/s41586-022-04417-6.

Correspondence and requests for materials should be addressed to Andrés Palencia or Malene Ringkjøbing Jensen.

Peer review information Nature thanks Hashim Al-Hashimi and the other, anonymous, reviewers for their contribution to the peer review of this work. Peer reviewer reports are available.

Reprints and permissions information is available at http://www.nature.com/reprints. 

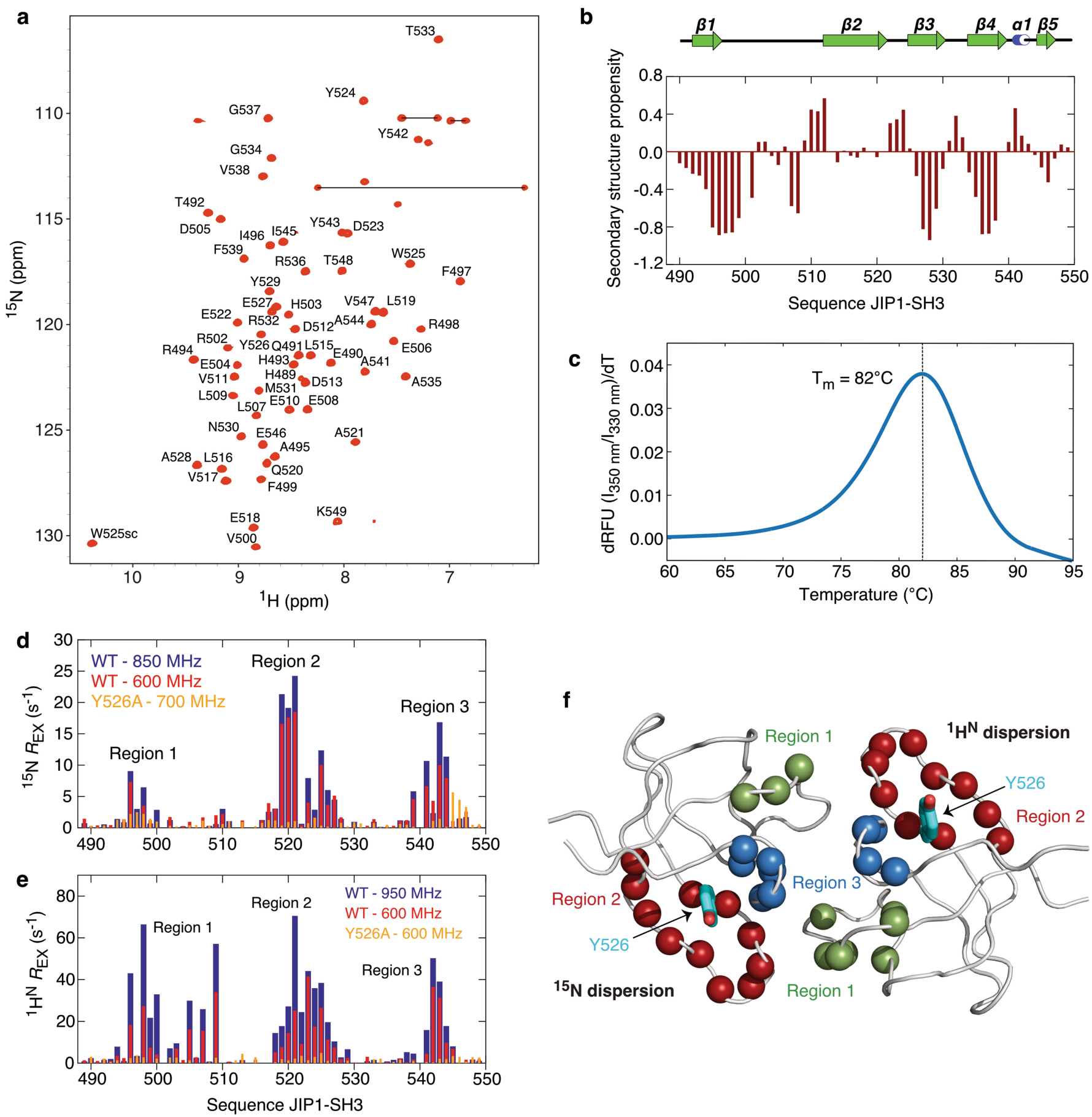

Extended Data Fig. 1 |Structural propensities and conformational exchange in JIP1-SH3. a, ${ }^{1} \mathrm{H}^{-15} \mathrm{~N} \mathrm{HSQC}$ spectrum of JIP1-SH 3 at $25^{\circ} \mathrm{C}$ with labels indicating assignments. Horizontal lines connect side chain resonances. b, Secondary structure propensities of JIP1-SH 3 calculated from experimental ${ }^{13} \mathrm{C} \alpha$ and ${ }^{13} \mathrm{C} \beta$ chemical shifts at $25^{\circ} \mathrm{C}$. The position of secondary structure elements is indicated, as observed in the crystal structure of JIP1-SH3 (PDB: 2FPE). c, Differential scanning fluorimetry (DSF) melting curve for JIP1-SH3 (RFU - relative fluorescence unit).d, Conformational exchange contributions, $R_{\mathrm{EX}}$, extracted from ${ }^{15} \mathrm{NCPMG}$ relaxation dispersion data acquired at $15^{\circ} \mathrm{C}$ of JIP1-SH3 (blue-850 MHz, red-600 MHz) andJIP1-SH3(Y526A) (orange - $700 \mathrm{MHz}$ )

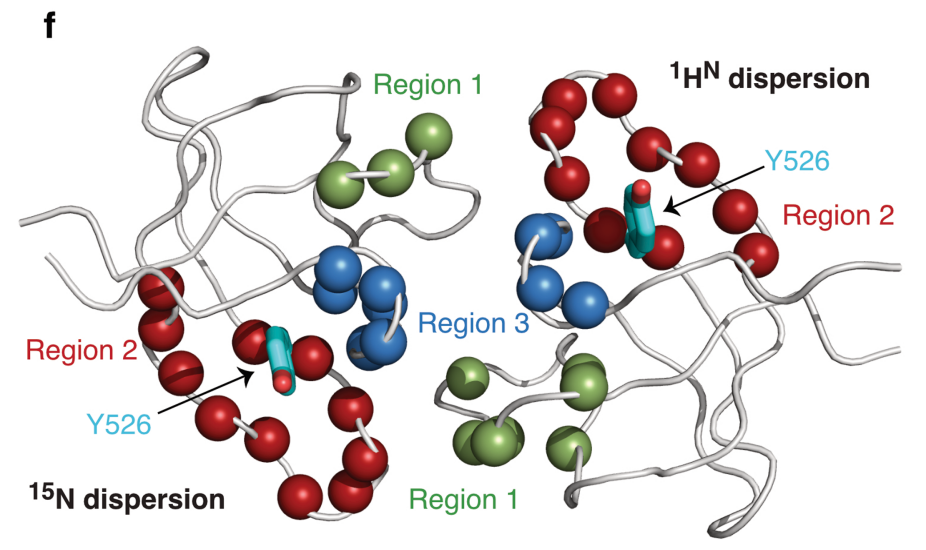

as the difference between $R_{2 \text { eff }}$ at low $(31 \mathrm{~Hz})$ and high $(1 \mathrm{kHz})$ CPMG frequencies.e, Exchange contributions, $R_{\mathrm{Ex}}$, extracted from ${ }^{1} \mathrm{H}^{\mathrm{N}} \mathrm{CPMG}$ relaxation dispersion data acquired at $15^{\circ} \mathrm{C}$ of JIP1-SH3 (blue $-950 \mathrm{MHz}$, red-600 MHz) and JIP1-SH3(Y526A) (orange $-600 \mathrm{MHz}$ ) as the difference between $R_{\text {2eff }}$ at low $(50 \mathrm{~Hz})$ and high $(2 \mathrm{kHz})$ CPMG frequencies. f, Structure of the dimeric SH3 domain of JIP1 with Y526 shown in cyan. Residues with exchange contributions $\left({ }^{15} \mathrm{~N} R_{\mathrm{EX}}>3 \mathrm{~s}^{-1}\right.$ and $\left.{ }^{1} \mathrm{H}^{\mathrm{N}} R_{\mathrm{EX}}>10 \mathrm{~s}^{-1}\right)$ are shown as spheres with colours indicating their position in the primary sequence (region 1 - green, region 2 - red, region 3 - blue). The residues showing ${ }^{15} \mathrm{~N}_{\text {and }}{ }^{1} \mathrm{H}^{\mathrm{N}}$ exchange contributions are represented separately on each monomer. 

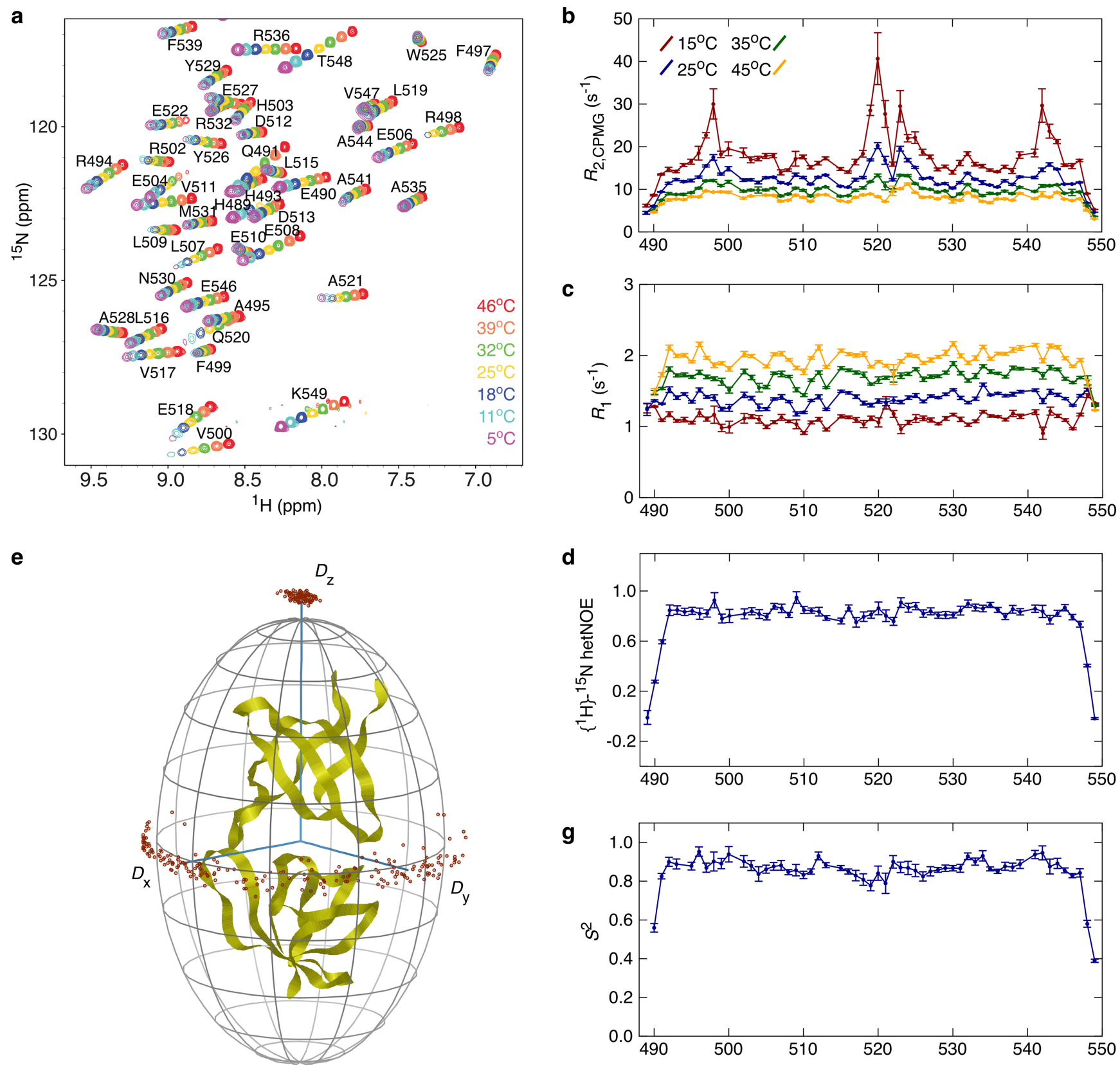

f
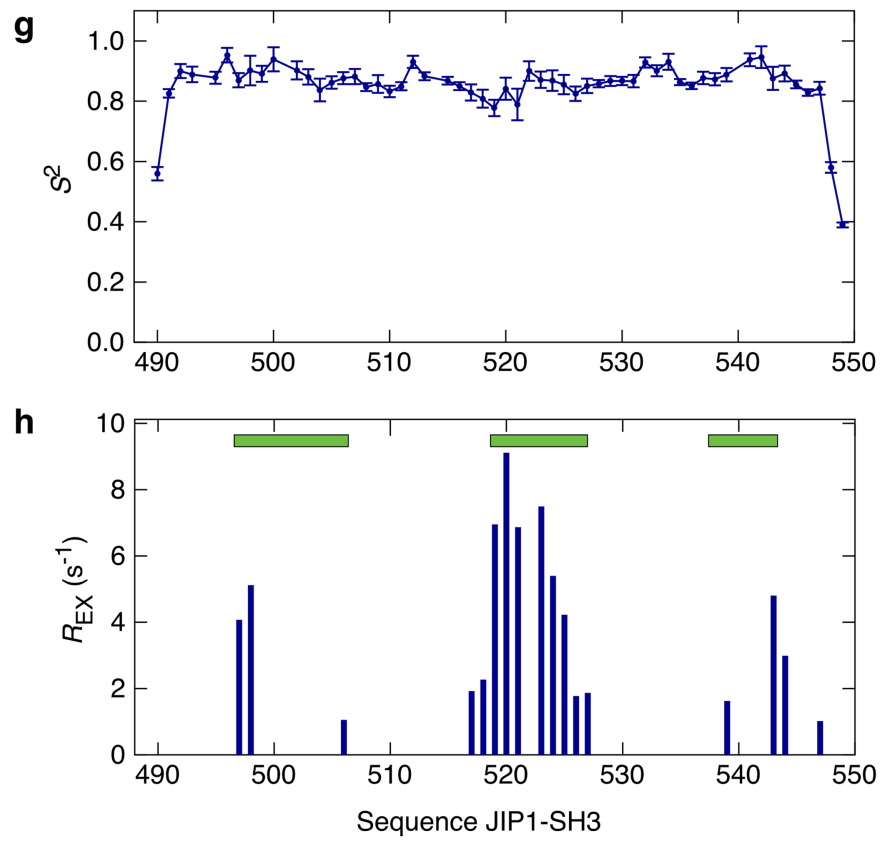

Extended Data Fig. 2 |See next page for caption. 


\section{Article}

Extended Data Fig. 2 |Lipari-Szabo model-free analysis of ${ }^{15} \mathrm{~N}$ relaxation data of JIP1-SH3. a, Region of the ${ }^{1} \mathrm{H}^{-15} \mathrm{~N}$ HSQC spectrum of JIP1-SH3 at temperatures ranging from 5 to $46^{\circ} \mathrm{C}$. b. Experimental ${ }^{15} \mathrm{~N} R_{2}(\mathrm{CPMG})$ relaxation rates at a ${ }^{1} \mathrm{H}$ frequency of $600 \mathrm{MHz}$ and four different temperatures. c, Experimental ${ }^{15} \mathrm{~N} R_{1}$ relaxation rates at a ${ }^{1} \mathrm{H}$ frequency of $600 \mathrm{MHz}$ and four different temperatures. d, Experimental $\left\{{ }^{1} \mathrm{H}\right\}{ }^{-15} \mathrm{~N}$ heteronuclear NOEs acquired at a ${ }^{1} \mathrm{H}$ frequency of $600 \mathrm{MHz}$ and $25^{\circ} \mathrm{C}$. Error bars in b-d represent one standard deviation (s.d.) derived from Monte Carlo simulations of experimental uncertainty.e, A model-free analysis of ${ }^{15} \mathrm{~N} R_{1}, R_{2}$ and heteronuclear NOEs at $25^{\circ} \mathrm{C}$ was carried out providing an axially symmetric diffusion tensor (Supplementary Discussion). The diffusion tensor is represented relative to the dimeric structure of JIP1-SH3. Distributions of axis orientations are shown as red dots and were determined from Monte Carlo simulations using Tensor $2^{75}$.f, Angular dependence of the $R_{2} / R_{1}$ ratios relative to the main axis of the diffusion tensor of JIP1-SH3. Only residues without exchange contributions to the transverse relaxation and for which the $\left\{{ }^{1} \mathrm{H}\right\}{ }^{15} \mathrm{~N}$ NOE is above 0.7 were included in the analysis. Error bars are centred at experimental values and were propagated from the experimental uncertainty on $R_{2}$ and $R_{1}$. Orange squares are back-calculated values using the optimal tensor. $g$, Order parameters, $S^{2}$, derived from the model-free analysis of the relaxation data at $25^{\circ} \mathrm{C}$. Error bars represent one standard deviation (s.d.) derived from Monte Carlo simulations as implemented in Tensor2.

h, Conformational exchange contributions, $R_{\mathrm{EX}}$, derived from the model-free analysis of the relaxation data at $25^{\circ} \mathrm{C}$. Green bars indicate residues that are located in the dimer interface of the $\mathrm{SH} 3$ domain as detected by the PISA server $^{76}$. 
a
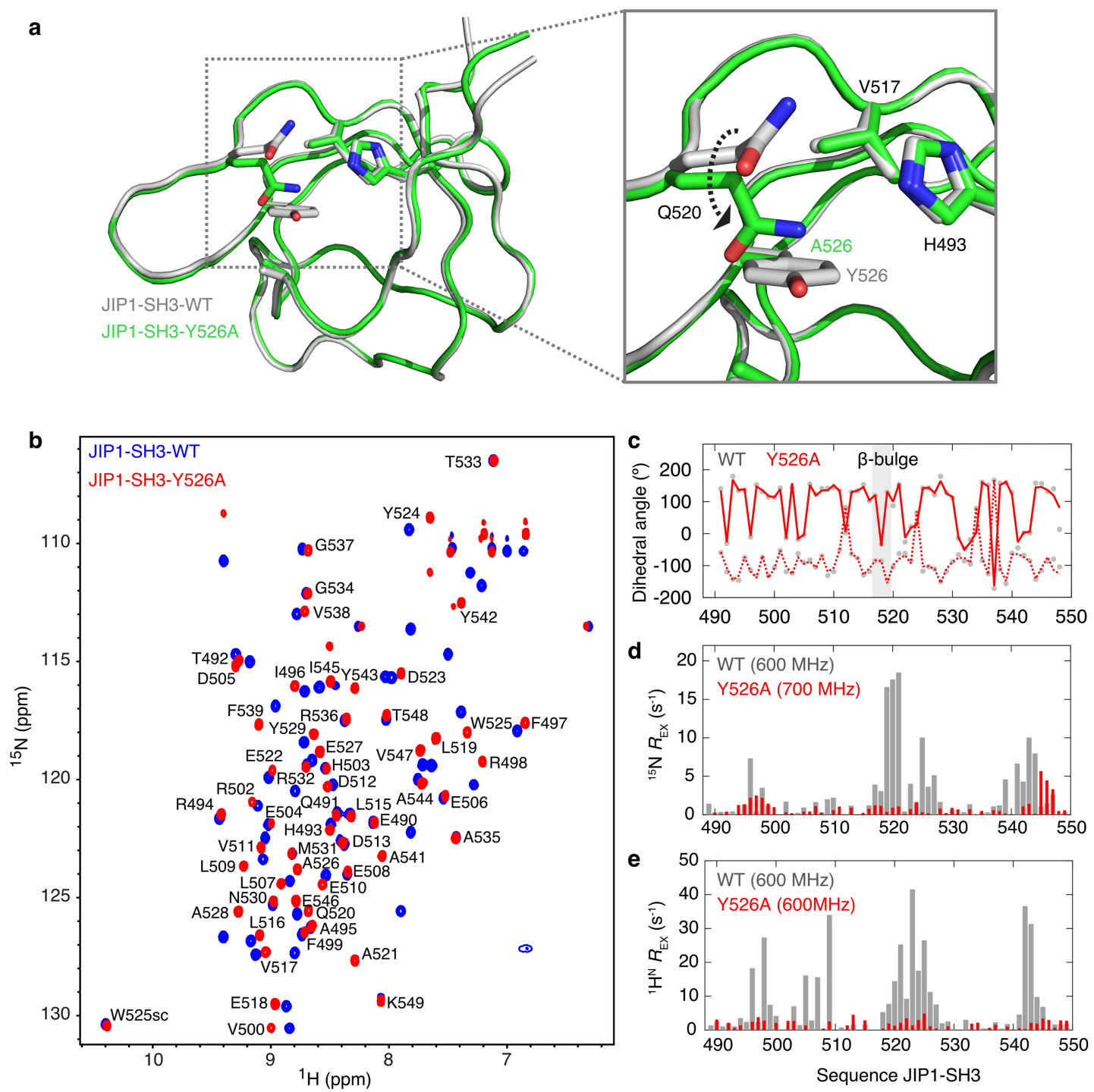

f

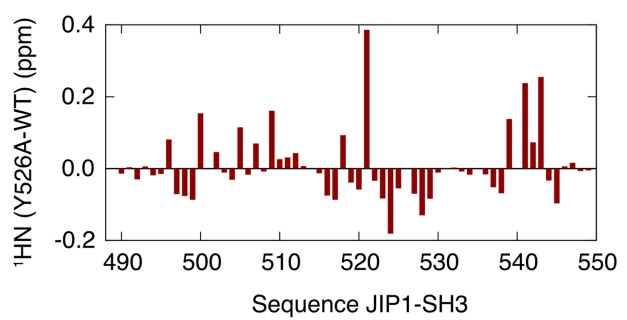

g

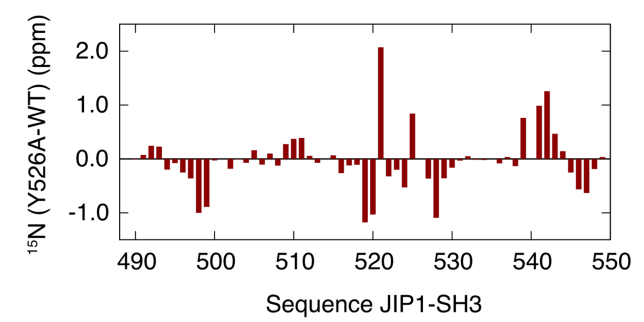

h

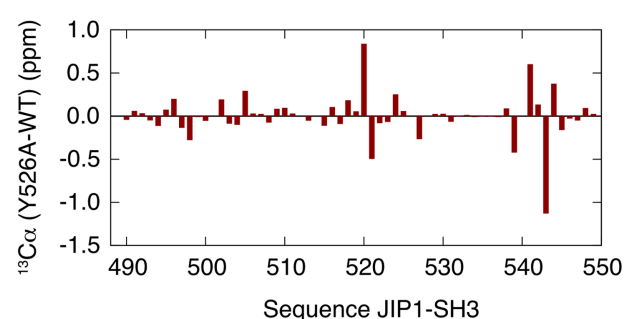

i

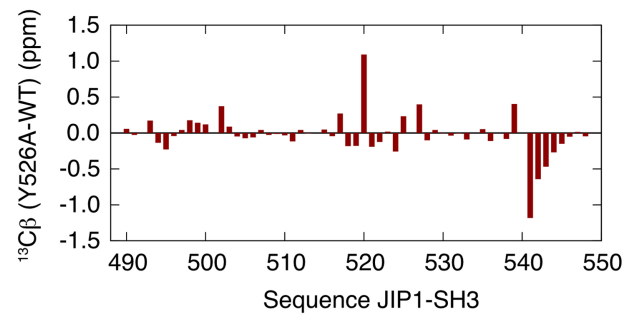

Extended Data Fig. 3 | See next page for caption. 


\section{Article}

Extended Data Fig. 3 |Structure and dynamics of the Y526A variant of JIP1-SH3. a, Comparison of the crystal structure of JIP1-SH3(Y526A) (green) with the WT structure (grey) showing an almost identical backbone conformation of the two proteins. The zoom highlights a minor structural difference at the level of the side chain of Q520 which reorients in the variant to take up the position normally occupied by Y526 in the WT protein. b, Superposition of ${ }^{1} \mathrm{H}^{-15} \mathrm{~N} \mathrm{HSQC}$ spectra of JIP1-SH3(Y526A) (red) and JIP1-SH3(WT) (blue) acquired at $25^{\circ} \mathrm{C}$. c, Comparison of dihedral angles in JIP1-SH3(WT) (grey spheres) and JIP1SH3(Y526A) (red lines). Dashed lines correspond to the backbone $\varphi$ angle and full drawn lines to the backbone $\psi$ angle. d, Conformational exchange contributions, $R_{\mathrm{Ex}}$, extracted from ${ }^{15} \mathrm{NCPMG}$ relaxation dispersion data as the difference between $R_{\text {2eff }}$ at low $(31 \mathrm{~Hz})$ and high $(1 \mathrm{kHz})$ CPMG frequencies. The exchange contributions are compared for WT JIP1-SH3 (grey, at $600 \mathrm{MHz}$ ) and the Y526A variant (red, at $700 \mathrm{MHz}$ ).e,Conformational exchange contributions, $R_{\mathrm{EX}}$, extracted from ${ }^{1} \mathrm{H}^{\mathrm{N}} \mathrm{CPMG}$ relaxation dispersion data as the difference between $R_{\text {2eff }}$ at low $(50 \mathrm{~Hz})$ and high $(2 \mathrm{kHz})$ CPMG frequencies. The exchange contributions are compared for the WT JIP1-SH3 (grey, at $600 \mathrm{MHz}$ ) and the Y526A variant (red, at $600 \mathrm{MHz}$ ). $\mathbf{f}-\mathbf{i}$, Chemical shift differences between the Y526A variant and WT JIP1-SH 3 for ${ }^{1} \mathrm{H}^{\mathrm{N}}(\mathbf{f}),{ }^{15} \mathrm{~N}(\mathbf{g}),{ }^{13} \mathrm{C} \alpha(\mathbf{h})$ and ${ }^{13} \mathrm{C} \beta(\mathbf{i})$. 

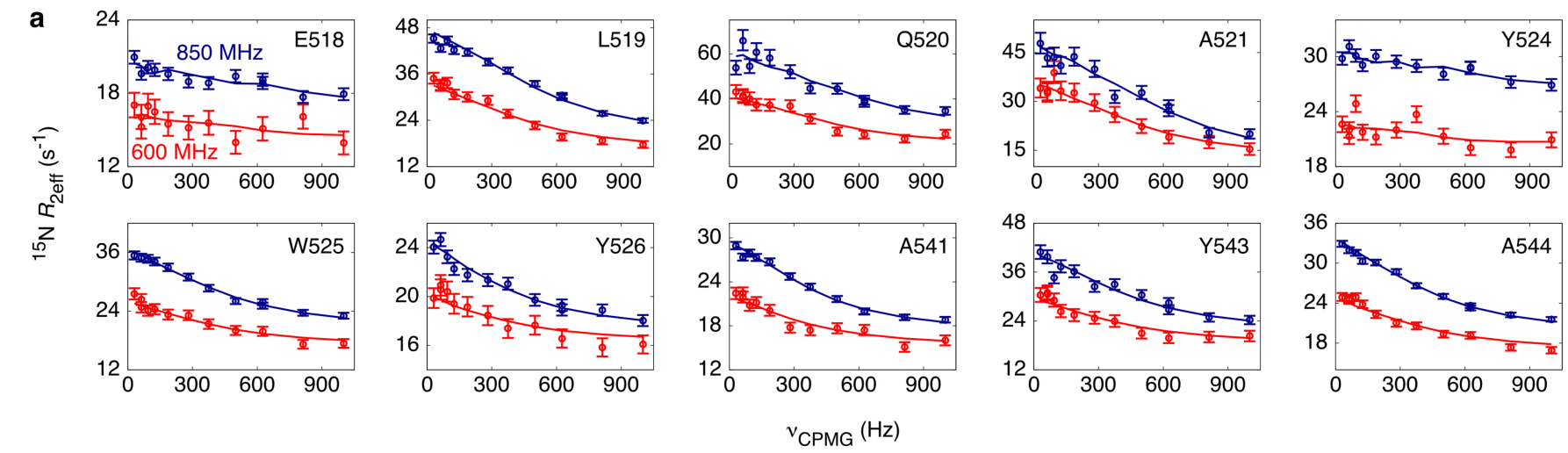

b
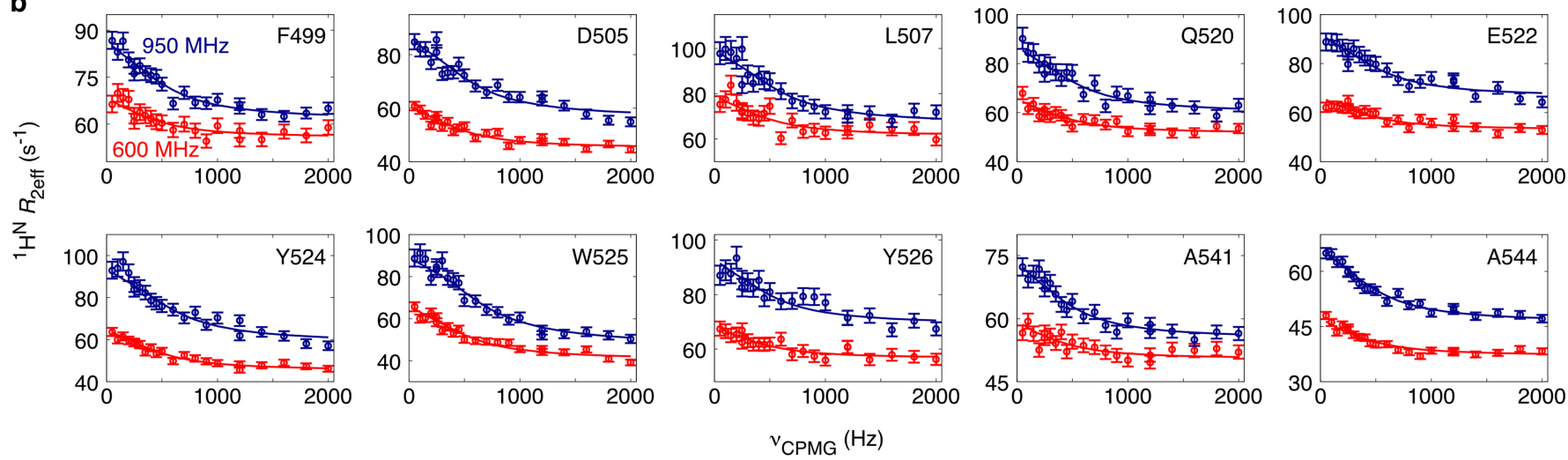

C

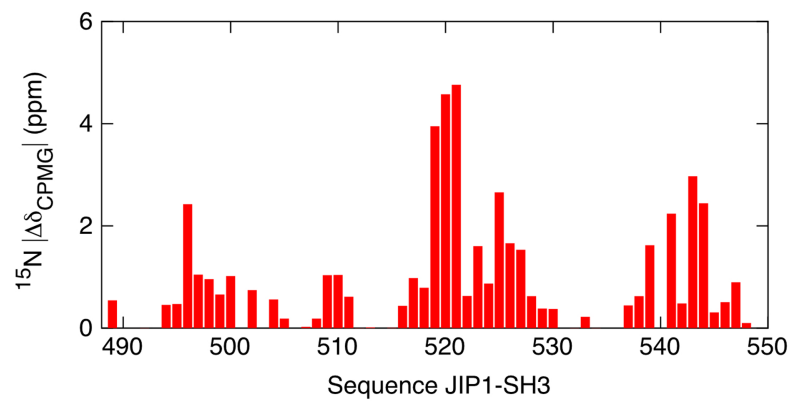

Extended Data Fig. 4 | Analysis of ${ }^{15} \mathrm{~N}$ and ${ }^{1} \mathrm{H}^{\mathrm{N}}$ relaxation dispersion data of JIP1-SH3. a, Examples of ${ }^{15} \mathrm{~N}$ CPMG relaxation dispersion profiles for JIP1-SH3 obtained at two magnetic field strengths (red $-600 \mathrm{MHz}$, blue $-850 \mathrm{MHz}$ ) at $15^{\circ} \mathrm{C}$. b. Examples of ${ }^{1} \mathrm{H}^{\mathrm{N}} \mathrm{CPMG}$ relaxation dispersion profiles for JIP1-SH3 obtained at two magnetic field strengths (red $-600 \mathrm{MHz}$, blue $-950 \mathrm{MHz}$ ) at $15^{\circ} \mathrm{C}$. The ${ }^{15} \mathrm{~N}$ and ${ }^{1} \mathrm{H}^{\mathrm{N}}$ data were analysed simultaneously for all residues d

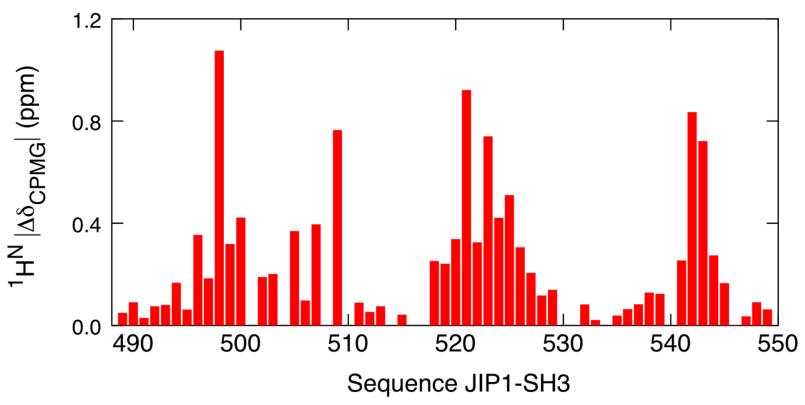

according to a two-site exchange model (full-drawn lines in $\mathbf{a}$ and $\mathbf{b}$ ). Error bars in $\mathbf{a}$ and $\mathbf{b}$ represent one standard deviation (s.d.) derived from Monte Carlo simulations of experimental uncertainty. c, d, Chemical shift differences between the major and minor state extracted from a simultaneous analysis of the relaxation dispersion data at $15^{\circ} \mathrm{C}$ for ${ }^{15} \mathrm{~N}(\mathbf{c})$ and ${ }^{1} \mathrm{H}^{\mathrm{N}}(\mathbf{d})$. 

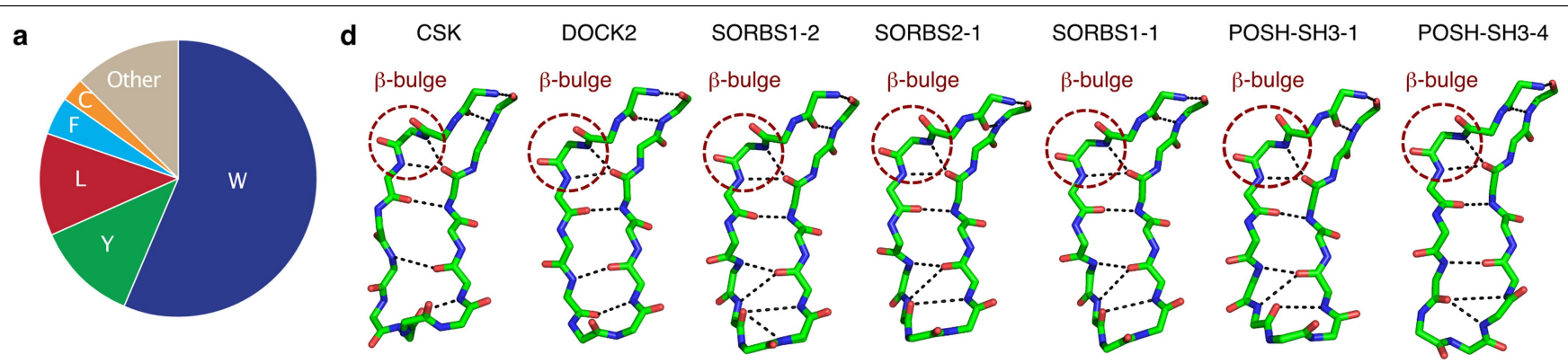

b

$\begin{array}{llc} & 490 & 500\end{array}$

PCA Crystal
group structure 2FPE $11.8 \AA$ $1 C S K / 2.5 \AA$ 7NZD/1.5A (This work)

C

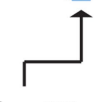

Position 493
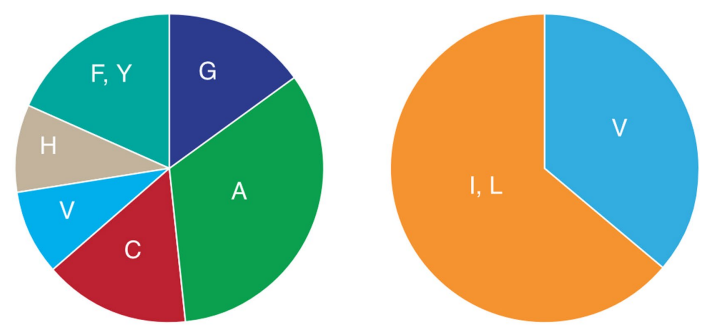

$n=0$
$n=1$

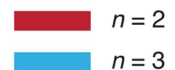

Extended Data Fig. 5 | Analysis of the sequence composition of 320 human SH3 domains. a, Distribution of amino acid types at the position of Y526 of JIP1-SH3 following a sequence alignment of all $320 \mathrm{SH} 3$ domains. b, Sequence alignment of all identified $\mathrm{SH} 3$ domains carrying either a phenylalanine $(\mathrm{F})$ or tyrosine (Y) at the position of Y526 in JIP1-SH3. For each sequence the PCA group is indicated along with the PDB code and resolution of available crystal structures. c, Pie charts showing the distribution of amino acid types in the different $\mathrm{SH} 3$ domains at positions corresponding to residue 493, 517,520 and

Position 520
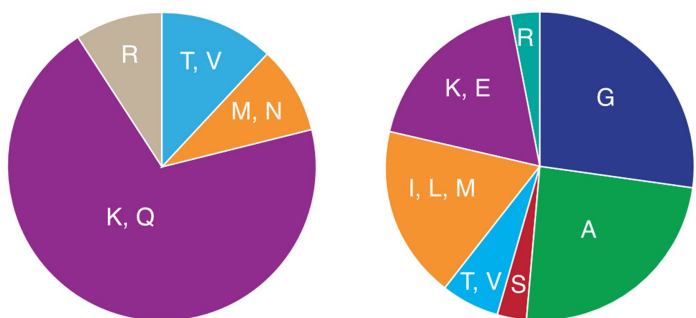

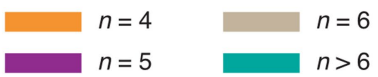

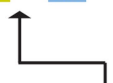

Position 541

541 in JIP1-SH3. The pie charts are colour-coded according to the size score assigned to each amino acid type corresponding to the number of heavy atoms in their side chains (see online Methods). d, Illustration of the backbone conformation of the $\beta$-sheet formed between the 516-521 and 524-529 regions in $\mathrm{SH} 3$ domains with tyrosine or phenylalanine at position 526 . The $\beta$-bulge conformation observed in WT JIP1-SH3 is observed in all SH3 domains for which high-resolution crystal structures are available. 

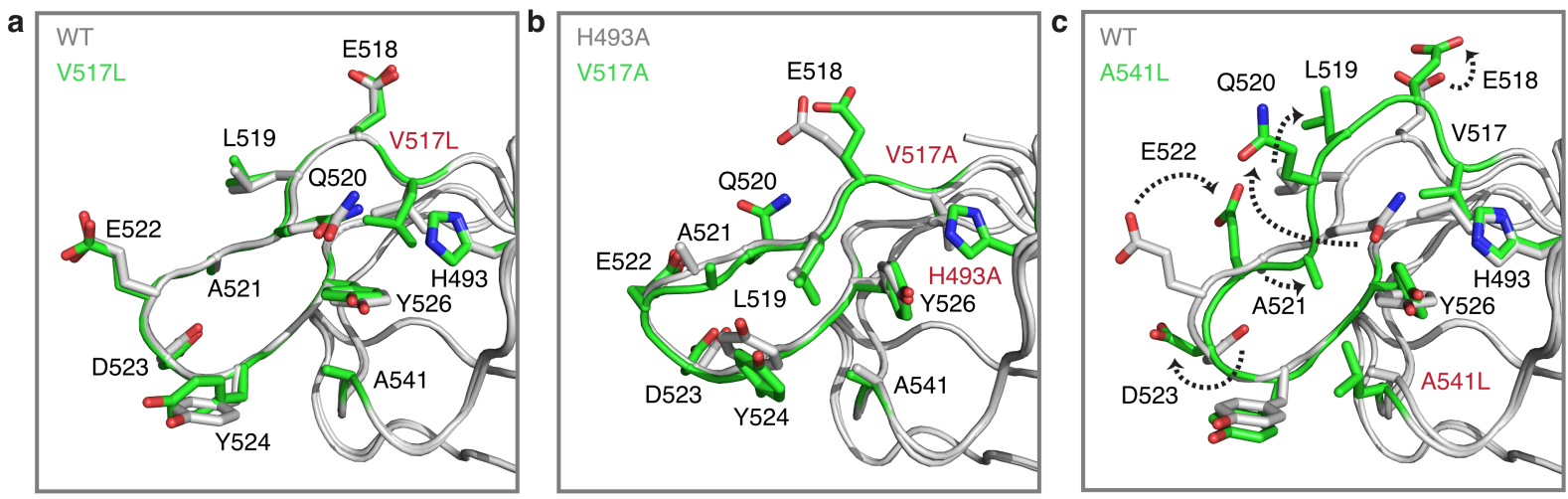

d
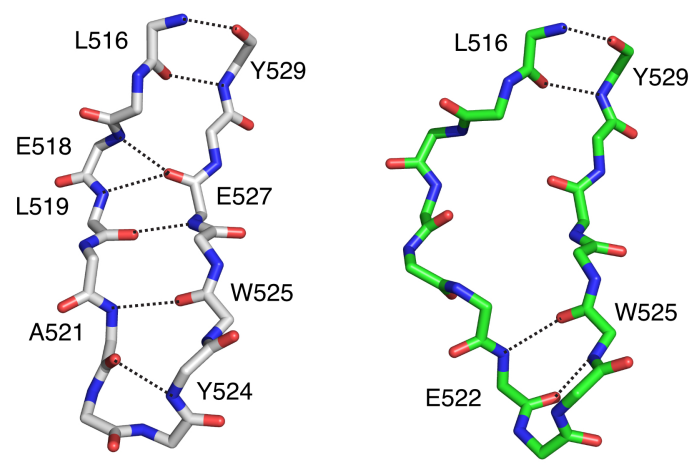

e

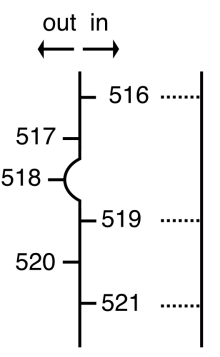

WT

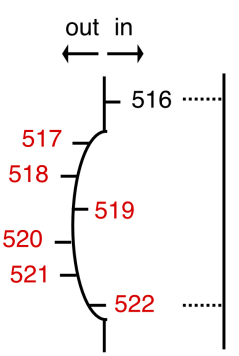

A541L

WT

A541L

f

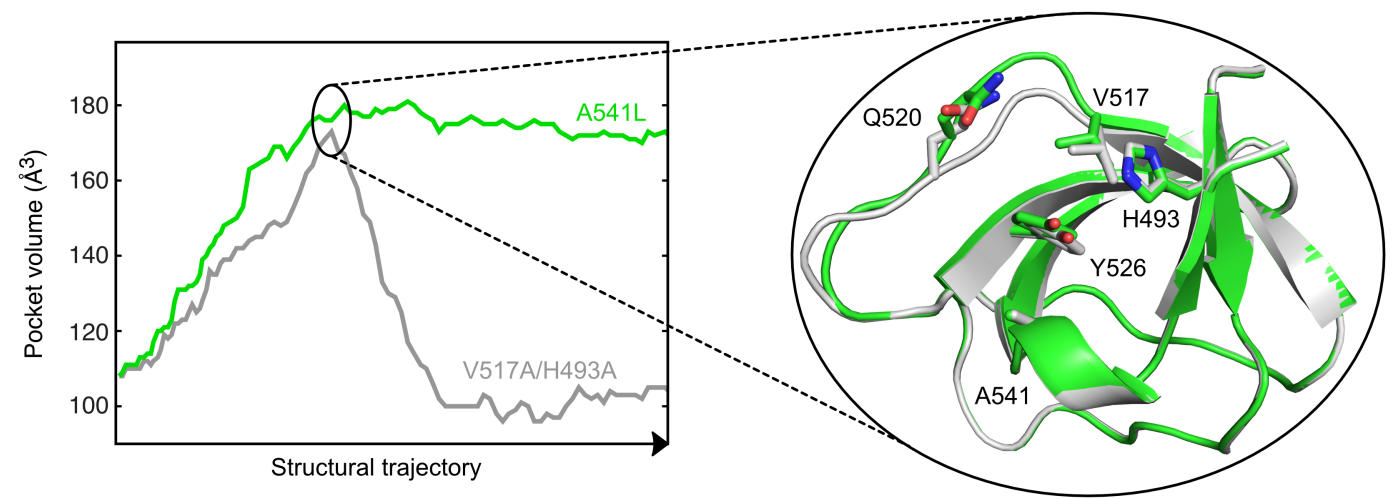

Extended Data Fig. 6 | Comparison of the structures of JIP1-SH3 and different variants. a, Comparison of the crystal structures of JIP1-SH3(WT) (grey) and its V517L variant (green). The backbone conformation is entirely conserved with only minor rearrangements of protein side chains.

b. Comparison of the crystal structures of the H493A (grey) and V517A (green) variants of JIP1-SH3. Structural features are conserved including similar side chain conformations. c, Comparison of the crystal structures of JIP1-SH3(WT) (grey) and its A541L variant (green) with arrows indicating the major conformational rearrangements between the WT protein and the variant. d, Illustration of the backbone conformation of the $\beta$-sheet formed between the 516-521 and 524-529 regions in the WT protein (left), and in the A541L variant (right). Dashed lines indicate hydrogen bonds. The A541L variant does not adopt a $\beta$-sheet conformation owing to the absence of several hydrogen bonds between the two $\beta$-strands. e, Schematic representation of the conformation of the $\beta$-strand encompassing residues 516 to 521 showing the orientation of the carbonyl group ("out" - carbonyl group surface exposed, "in" - carbonyl group pointing towards the $\beta$-strand encompassing residues 524 to 529) in the WT protein (left) and in the A541L variant (right).f, Volume of the Y526 pocket along the structural trajectory between the WT conformation and the conformations of the H493A/V517A mutants (grey) or A541L mutant (green). Conformations from the two structural trajectories at the maximum pocket volume are superimposed and shown in cartoon representation. The initial pocket expansion and its associated structural features are shared among all mutants. 
a

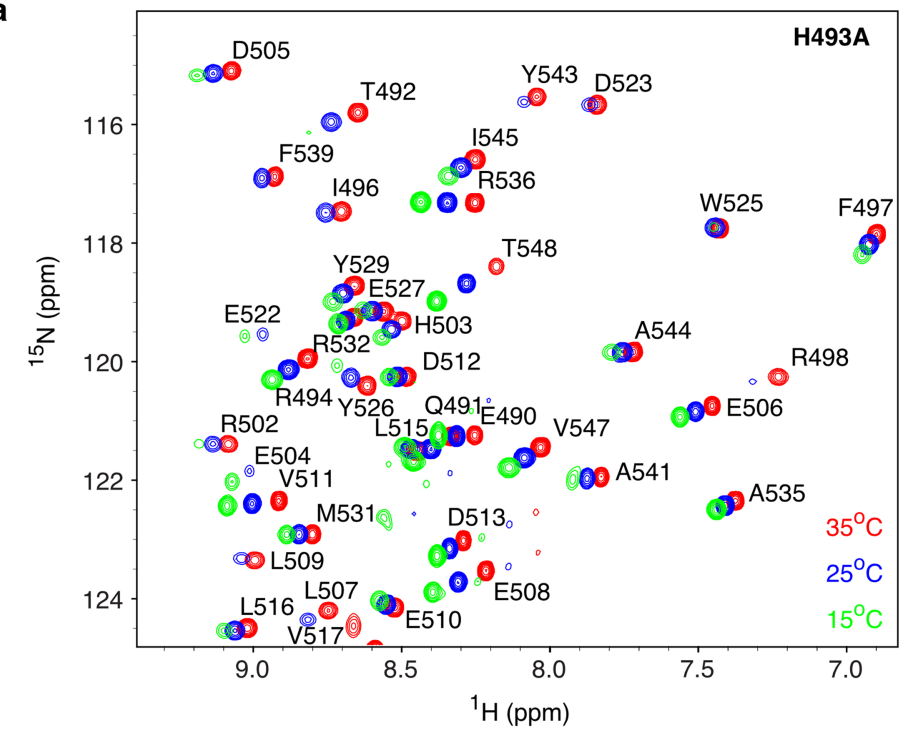

b

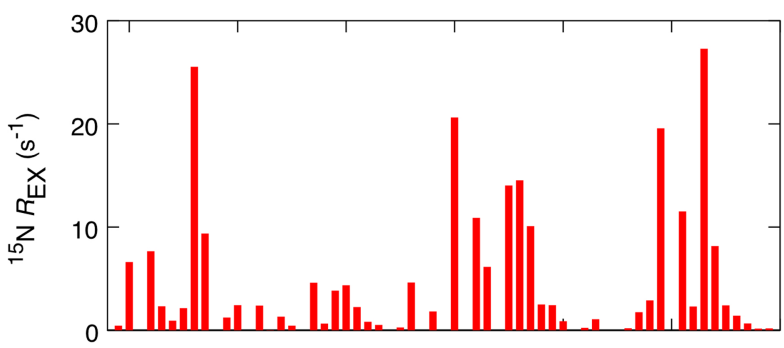

c

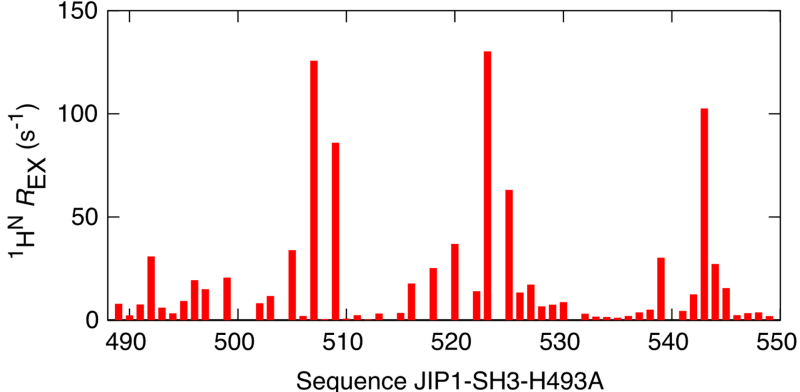

d

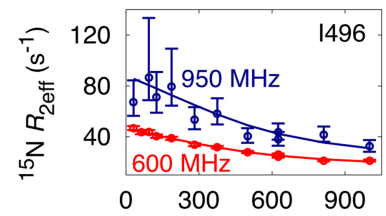

e

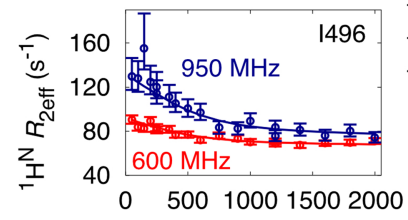

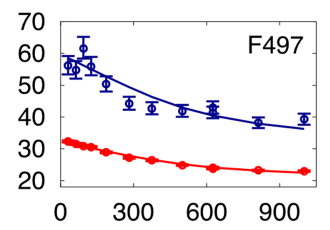

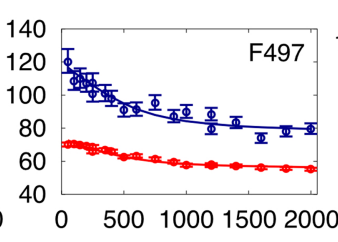

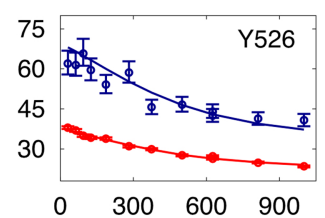
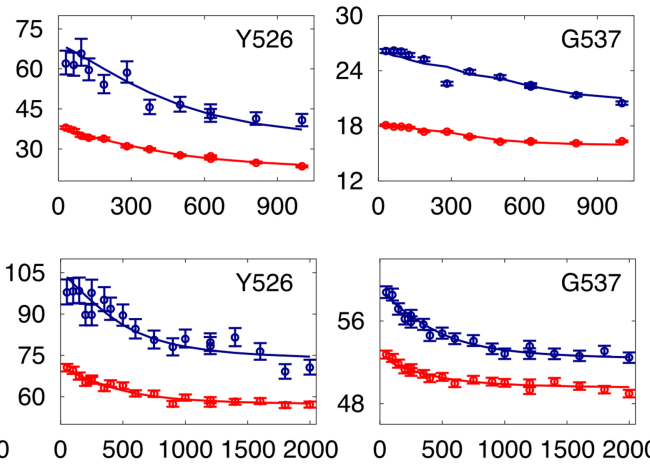

G537
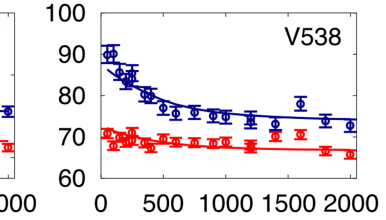

$v_{\mathrm{CPMG}}(\mathrm{Hz})$

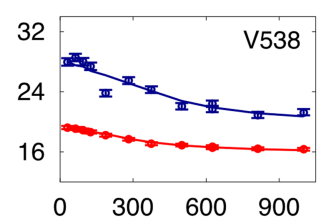

f

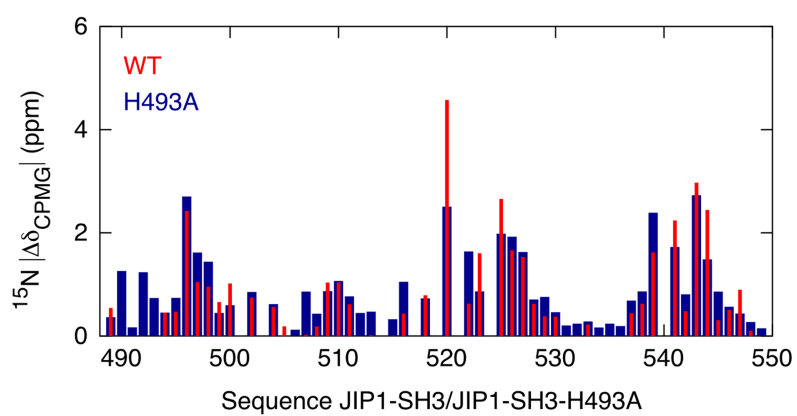

Extended Data Fig. 7 | Analysis of CPMG relaxation dispersion data of the H493A variant of JIP1-SH3. a, Region of the ${ }^{1} \mathrm{H}^{-15} \mathrm{~N} \mathrm{HSQC}$ of the $\mathrm{H} 493 \mathrm{~A}$ variant at three different temperatures (green $-15^{\circ} \mathrm{C}$, blue $-25^{\circ} \mathrm{C}$ and red $-35^{\circ} \mathrm{C}$ ). b, Conformational exchange contributions, $R_{\mathrm{Ex}}$, extracted from ${ }^{15} \mathrm{NCPMG}$ relaxation dispersion data of the $\mathrm{H} 493 \mathrm{~A}$ variant as the difference between $R_{2 \text { eff }}$ at low $(31 \mathrm{~Hz})$ and high $(1 \mathrm{kHz}) \mathrm{CPMG}$ frequencies $\left(600 \mathrm{MHz}\right.$ and $\left.15^{\circ} \mathrm{C}\right)$. c, Conformational exchange contributions, $R_{\mathrm{Ex}}$, extracted from ${ }^{1} \mathrm{H}^{\mathrm{N}} \mathrm{CPMG}$ relaxation dispersion data of the $\mathrm{H} 493 \mathrm{~A}$ variant as the difference between $R_{2 \text { eff }}$ at low $(50 \mathrm{~Hz})$ and high $(2 \mathrm{kHz}) \mathrm{CPMG}$ frequencies $\left(600 \mathrm{MHz}\right.$ and $\left.15^{\circ} \mathrm{C}\right)$. d, e, Examples of ${ }^{15} \mathrm{~N}(\mathbf{d})$ and ${ }^{1} \mathrm{H}^{\mathrm{N}}(\mathbf{e}) \mathrm{CPMG}$ relaxation dispersion profiles of the g

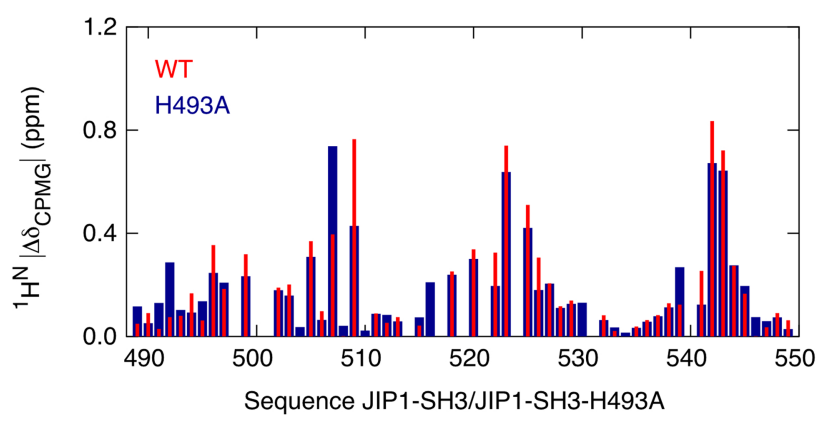

H493A variant of JIP1-SH3 obtained at two magnetic field strengths (red-600 MHz, blue $-950 \mathrm{MHz}$ ) at $15^{\circ} \mathrm{C}$. The ${ }^{15} \mathrm{~N}$ and ${ }^{1} \mathrm{H}^{\mathrm{N}}$ data were analysed simultaneously for all residues according to a two-site exchange model (full-drawn lines in $\mathbf{d}$ and e) using a population of the minor state fixed to $10 \%$. Error bars represent one standard deviation (s.d.) derived from Monte Carlo simulations of experimental uncertainty. $\mathbf{f}, \mathbf{g}$, Comparison of the chemical shift differences between the major and minor state extracted from relaxation dispersion experiments for WT JIP1-SH3 (red) and its H493A variant (blue). Data are shown for both ${ }^{15} \mathrm{~N}(\mathbf{f})$ and ${ }^{1} \mathrm{H}^{\mathrm{N}}(\mathbf{g})$ chemical shifts. 

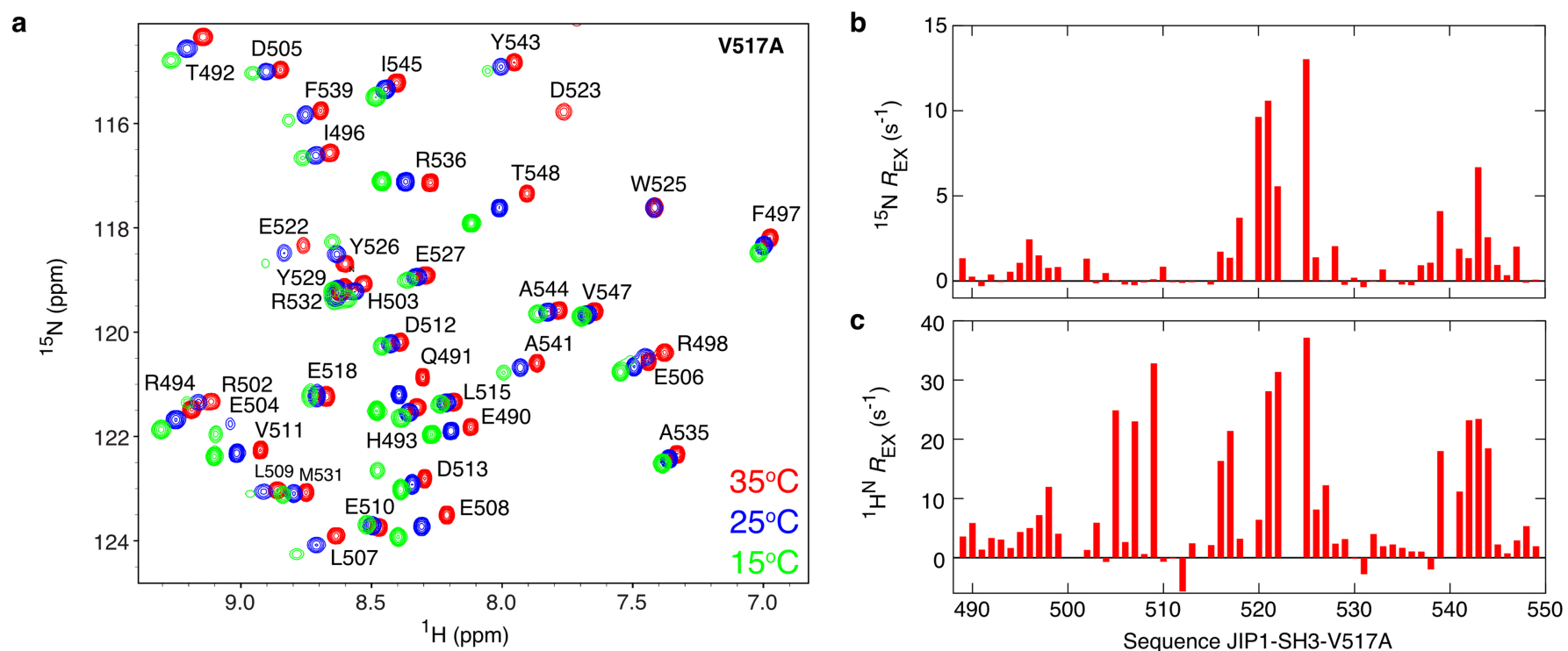

d
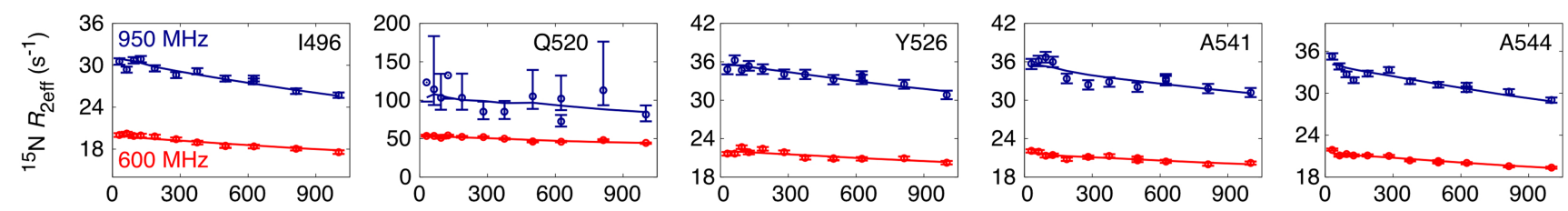

e
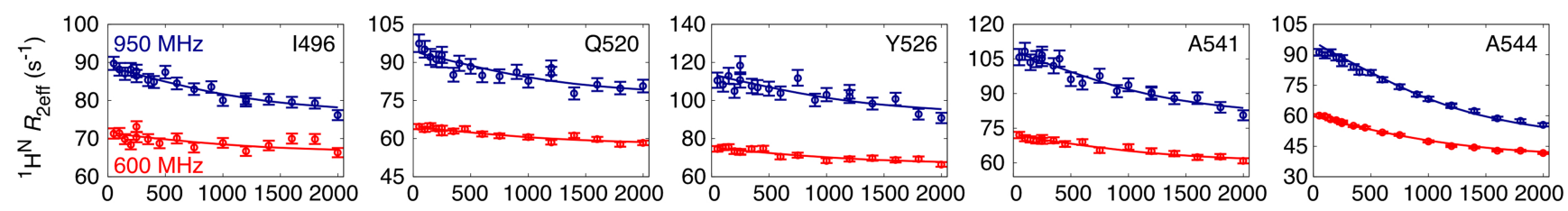

$v_{\text {CPMG }}(\mathrm{Hz})$

f

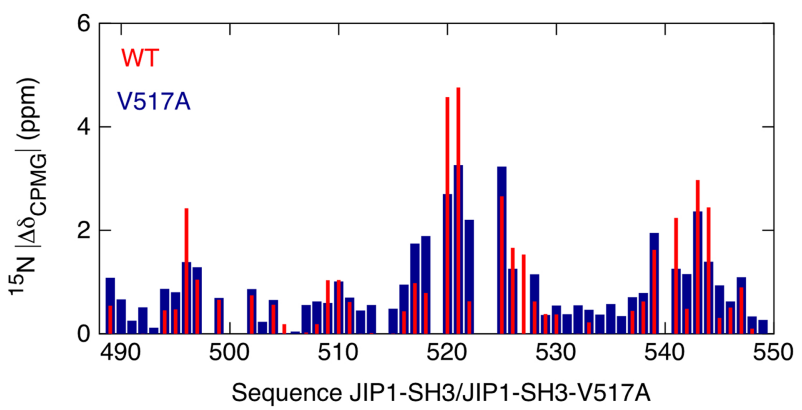

Extended Data Fig. 8 | Analysis of CPMG relaxation dispersion data of the V517A variant of JIP1-SH3. a, Region of the ${ }^{1} \mathrm{H}^{-15} \mathrm{~N} \mathrm{HSQC}$ of the V517A variant at three different temperatures (green $-15^{\circ} \mathrm{C}$, blue $-25^{\circ} \mathrm{C}$ and red $-35^{\circ} \mathrm{C}$ ). b, Conformational exchange contributions, $R_{\mathrm{Ex}}$, extracted from ${ }^{15} \mathrm{NCPMG}$ relaxation dispersion data of the V517A variant as the difference between $R_{2 \mathrm{eff}}$ at low $(31 \mathrm{~Hz})$ and high $(1 \mathrm{kHz}) \mathrm{CPMG}$ frequencies $\left(600 \mathrm{MHz}\right.$ and $\left.15^{\circ} \mathrm{C}\right) . \mathrm{c}$, Conformational exchange contributions, $R_{\mathrm{Ex}}$, extracted from ${ }^{1} \mathrm{H}^{\mathrm{N}} \mathrm{CPMG}$ relaxation dispersion data of the V517A variant as the difference between $R_{2 \text { eff }}$ at low $(50 \mathrm{~Hz})$ and high $(2 \mathrm{kHz}) \mathrm{CPMG}$ frequencies $\left(600 \mathrm{MHz}\right.$ and $\left.15^{\circ} \mathrm{C}\right)$. d, e, Examples of ${ }^{15} \mathrm{~N}(\mathbf{d})$ and ${ }^{1} \mathrm{H}^{\mathrm{N}}(\mathbf{e}) \mathrm{CPMG}$ relaxation dispersion profiles of the V517A g

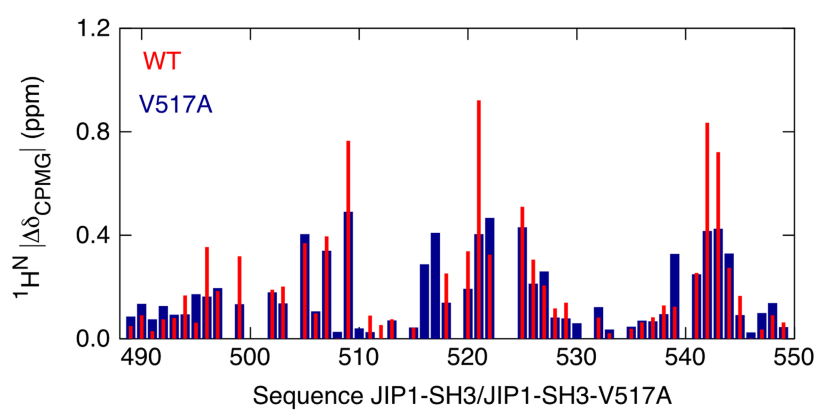

variant of JIP1-SH3 obtained at two magnetic field strengths (red $-600 \mathrm{MHz}$, blue $-950 \mathrm{MHz}$ ) at $15^{\circ} \mathrm{C}$. The ${ }^{15} \mathrm{~N}$ and ${ }^{1} \mathrm{H}^{\mathrm{N}}$ data were analysed simultaneously for all residues according to a two-site exchange model (full-drawn lines in $\mathbf{d}$ and $\mathbf{e}$ ) using a population of the minor state fixed to $11 \%$. Error bars represent one standard deviation (s.d.) derived from Monte Carlo simulations of experimental uncertainty. $\mathbf{f}, \mathbf{g}$, Comparison of the chemical shift differences between the major and minor state extracted from relaxation dispersion experiments for WT JIP1-SH3 (red) and its V517A variant (blue). Data are shown for both ${ }^{15} \mathrm{~N}(\mathbf{f})$ and ${ }^{1} \mathrm{H}^{\mathrm{N}}(\mathbf{g})$ chemical shifts. 


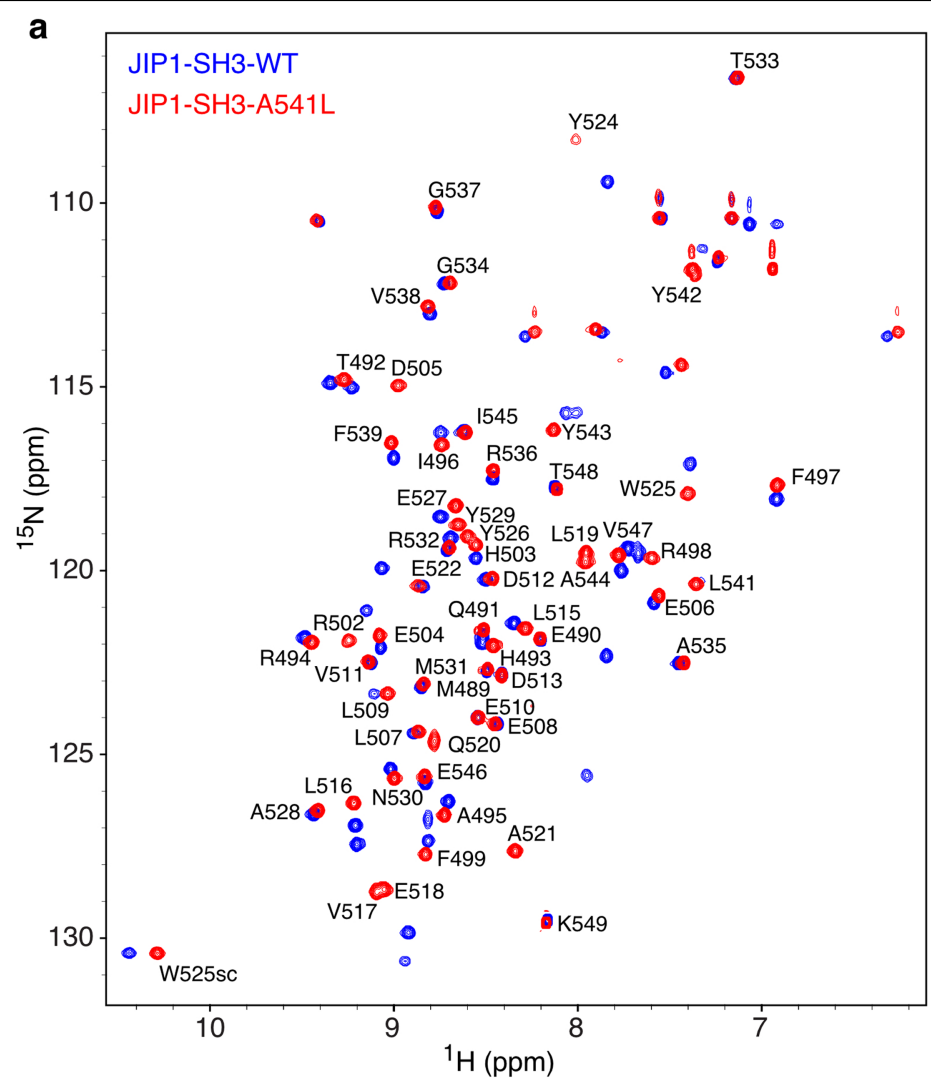

b

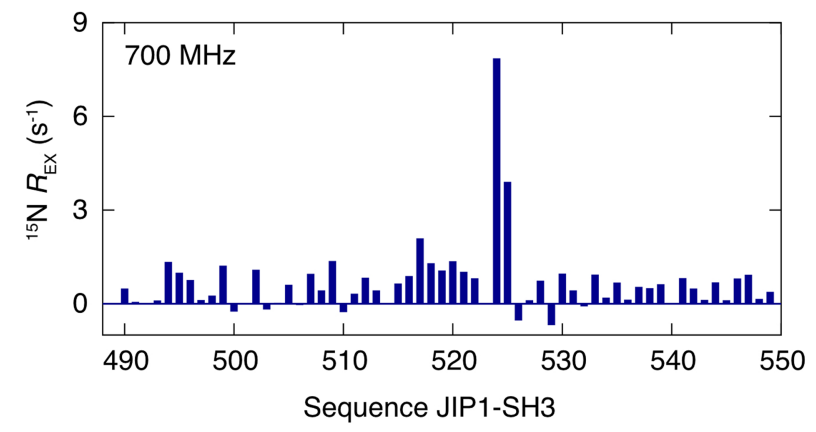

Extended Data Fig. 9 | The A541L variant does not capture the structural details of the minor state detected by CPMG relaxation dispersion in JIP1-SH3. a, Superposition of ${ }^{1} \mathrm{H}^{-15} \mathrm{~N}$ HSQC spectra of JIP1-SH3(A541L) (red) and WTJIP1-SH3 (blue) acquired at $15^{\circ} \mathrm{C} . \mathbf{b}$, Conformational exchange contributions, $R_{\mathrm{EX}}$, extracted from ${ }^{15} \mathrm{~N}$ CPMG relaxation dispersion data of the A541 variant as the difference between $R_{\text {2eff }}$ at low $(31 \mathrm{~Hz})$ and high $(1 \mathrm{kHz})$ CPMG frequencies at $700 \mathrm{MHz}$ and $15^{\circ} \mathrm{C}$. Only modest ${ }^{15} \mathrm{~N}$ conformational exchange contributions are observed suggesting that this variant is populating a single conformation in solution represented by the determined crystal
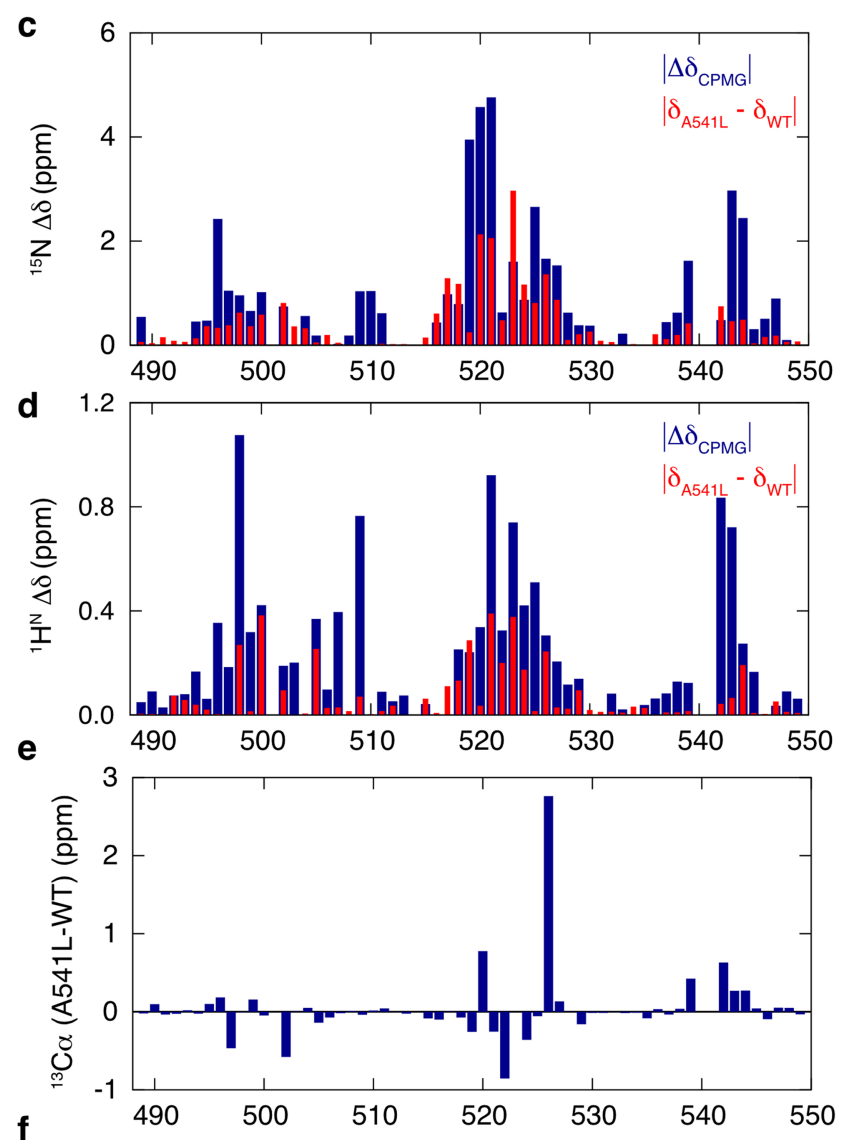

f

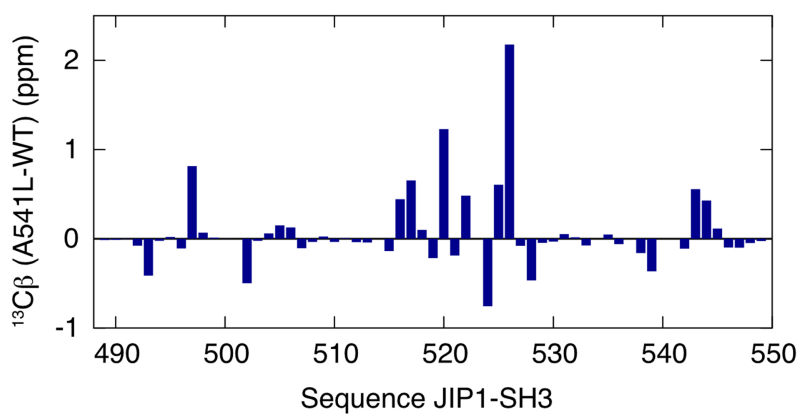

structure.c, d, Comparison of the chemical shift differences between the major and minor states extracted from relaxation dispersion experiments of WT JIP1-SH3 (blue) and the chemical shift differences between the observed chemical shifts of the A541L variant and WT JIP1-SH3 (red). Data are shown for ${ }^{15} \mathrm{~N}(\mathbf{c})$ and for ${ }^{1} \mathrm{H}^{\mathrm{N}}(\mathbf{d})$ nuclei. The poor agreement between the two datasets show that the A541L crystal structure is not representative of the conformation of the minor state detected by NMR relaxation dispersion.e, $\mathbf{f}$, Chemical shift differences between the A541L variant and WT JIP1-SH3 for ${ }^{13} \mathrm{C} \alpha(\mathbf{e})$ and ${ }^{13} \mathrm{C} \beta(\mathbf{f})$. 

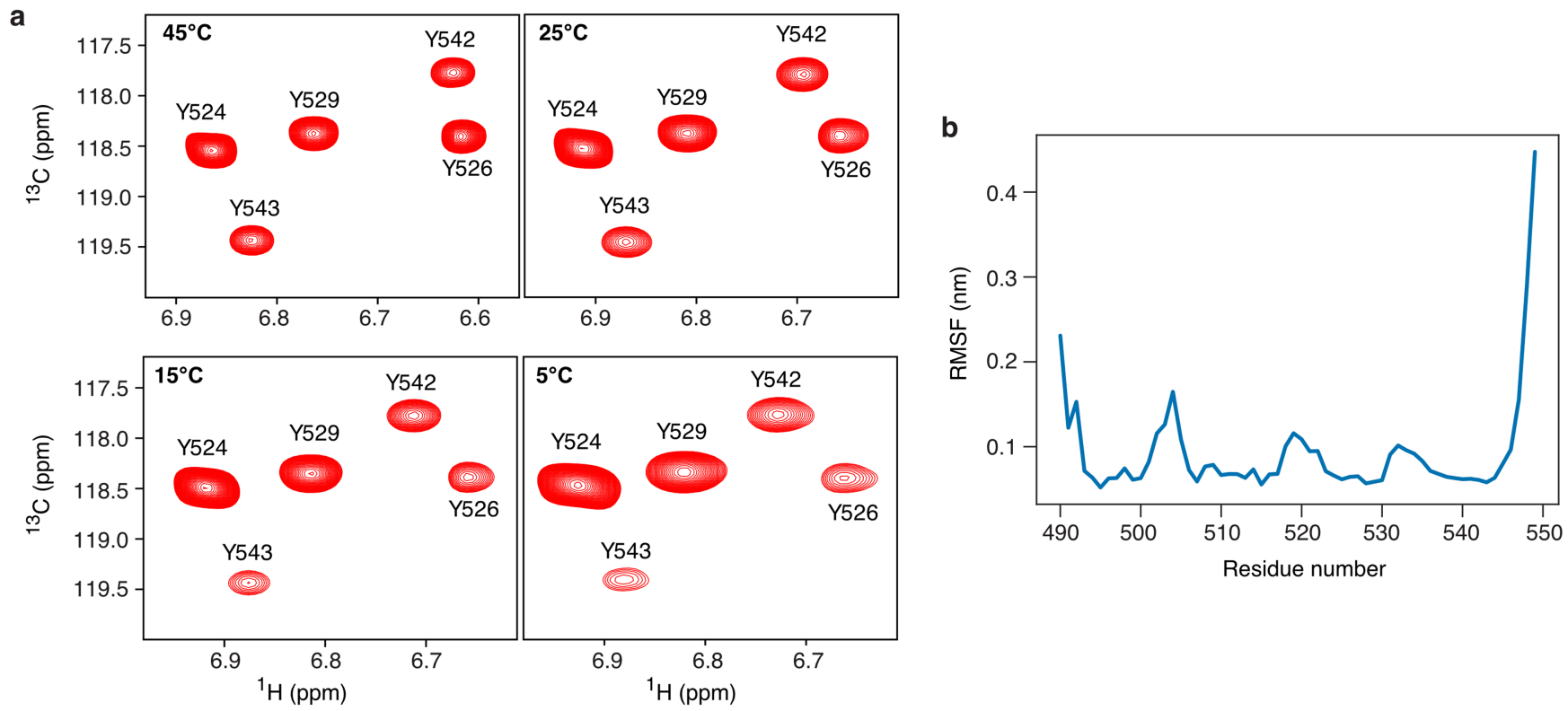

C
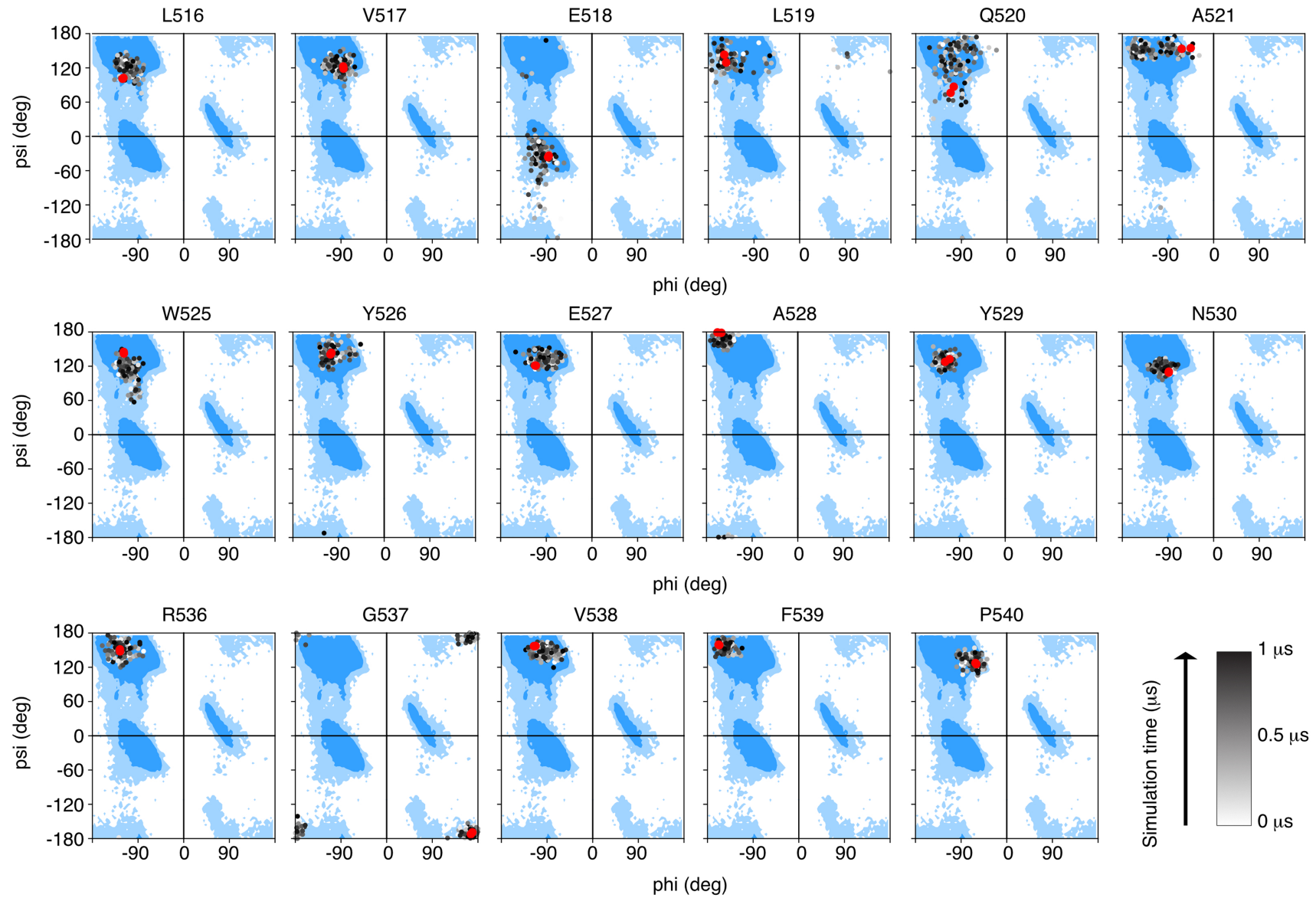

Extended Data Fig. 10 | The aromatic ring of Y526 undergoes fast flipping. a, ${ }^{1} \mathrm{H}^{13} \mathrm{CHSQC}$ spectra of JIP1-SH3 showing the region of tyrosine epsilon correlations. A sample of JIP1-SH3 site-selectively labelled at the epsilon positions was used, and spectra at four different temperatures were acquired in the range from 5 to $45^{\circ} \mathrm{C}$. b, Per-residue root-mean square fluctuation (RMSF) of $\mathrm{C} \alpha$ atoms during the MD simulation. c, Residue-specific Ramachandran plots showing the conformational sampling of $\beta$-strand residues during the MD simulation. The MD values are colour-coded from white to black according to simulation time. Blue regions indicate the allowed and marginally allowed regions. Red points indicate the starting conformation (one for each monomer of JIP1-SH3). Panels $\mathbf{b}$ and $\mathbf{c}$ demonstrate that the protein maintains a stable conformation throughout the MD simulation, while displaying several $180^{\circ}$ ring flipping events of Y526 (Fig. 4d). 


\section{Article}

Extended Data Table 1 | Data collection and refinement statistics for the JIP1-SH3 structures

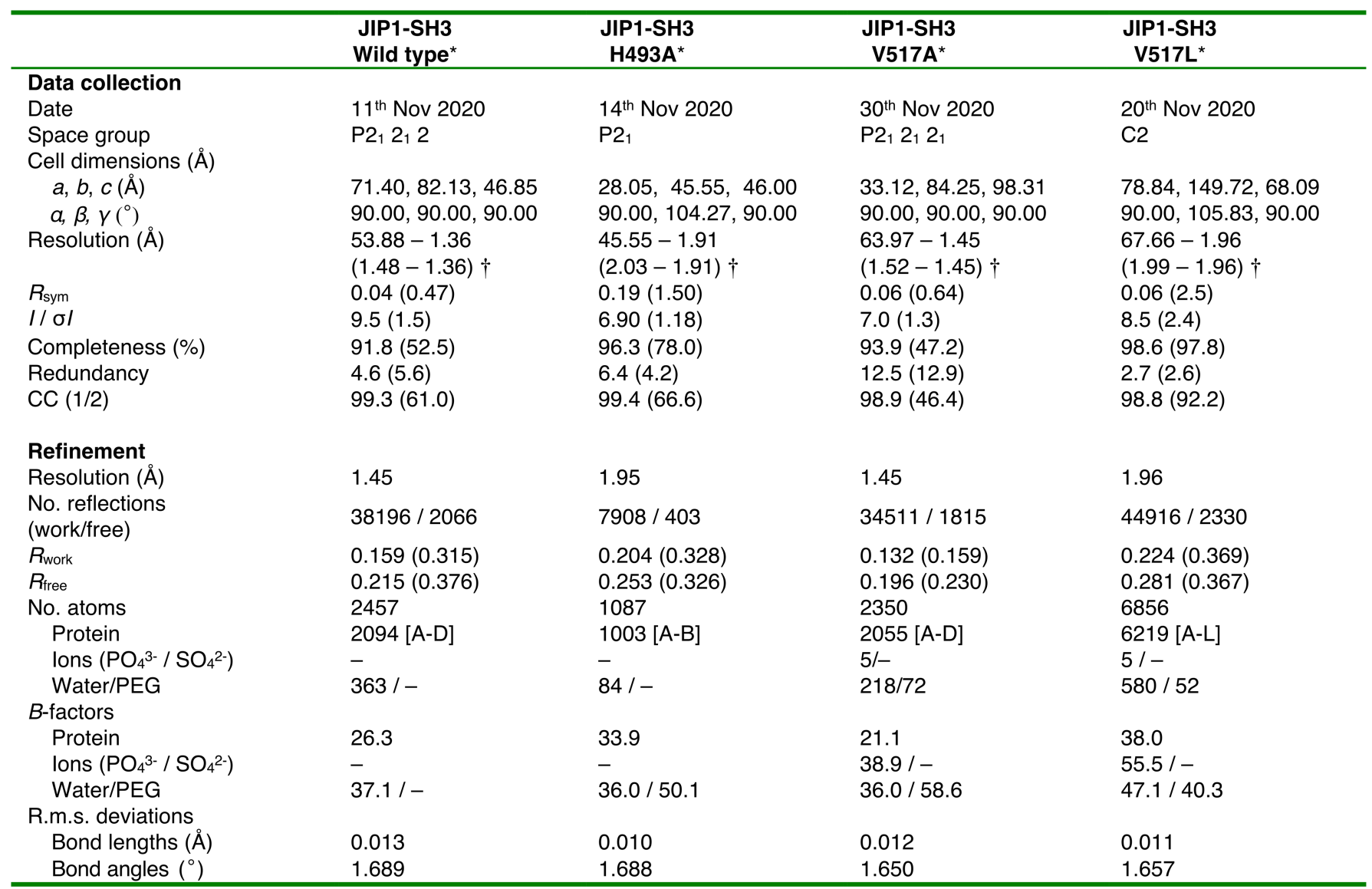

*Number of crystals for each structure: 1 .

+Values in parenthesis are for the highest-resolution shell. 
Extended Data Table 2 | Data collection and refinement statistics for the JIP1-SH3 and POSH-SH3 structures

\begin{tabular}{|c|c|c|c|c|}
\hline & $\begin{array}{c}\text { JIP1-SH3 } \\
\text { A541L* }^{*}\end{array}$ & $\begin{array}{c}\text { JIP1-SH3 } \\
\text { Y526A* }\end{array}$ & POSH-SH3-1* & POSH-SH3-4* \\
\hline Date & $30^{\text {th }}$ Oct 2020 & $5^{\text {th }}$ Dec 2020 & $1^{\text {st }}$ Aug 2019 & $17^{\text {th }}$ Feb 2020 \\
\hline \multicolumn{5}{|l|}{ Cell dimensions $(\AA)$} \\
\hline$a, b, c(\AA)$ & $96.09,67.10,59.14$ & $210.64,62.49,87.06$ & $35.96,35.96,181.33$ & $45.52,45.52,65.74$ \\
\hline$a, \beta, \gamma\left(^{\circ}\right)$ & $90.00,126.70,90.00$ & $90.00,90.00,90.00$ & $90.00,90.00,120.00$ & $90.00,90.00,120.00$ \\
\hline$R_{\mathrm{sym}}$ & $0.081(0.36)$ & $0.07(2.1)$ & $0.01(0.4)$ & $\begin{array}{l}(1.48-1.45) \\
0.01(0.7)\end{array}$ \\
\hline$|/ \sigma|$ & $5.5(1.5)$ & $11.9(0.8)$ & $26.1(1.4)$ & $23.8(0.9)$ \\
\hline Completeness (\%) & $91.9(52.1)$ & $99.9(98.7)$ & $93.0(89.9)$ & $99.9(99.2)$ \\
\hline Redundancy & $5.3(5.0)$ & $67.7(6.4)$ & $25.4(1.2)$ & $10.1(8.5)$ \\
\hline $\mathrm{CC}(1 / 2)$ & $98.2(74.0)$ & $99.9(30.7)$ & $0.99(0.57)$ & $0.99(0.52)$ \\
\hline$R_{\text {work }}$ & $0.194(0.328)$ & $0.138(0.361)$ & $0.146(0.282)$ & $0.160(0.489)$ \\
\hline$R_{\text {free }}$ & $0.281(0.408)$ & $0.179(0.379)$ & $0.177(0.273)$ & $0.227(0.655)$ \\
\hline No. atoms & 2638 & 7091 & 685 & 637 \\
\hline Protein & $2142[A-D]$ & $6129[\mathrm{~A}-\mathrm{L}]$ & 531 & 531 \\
\hline lons $\left(\mathrm{PO}_{4}{ }^{3-} / \mathrm{SO}_{4}{ }^{2-}\right)$ & - & $10 / 30$ & - & - \\
\hline Water/PEG & 522 & 915 & $139 / 15$ & $95 / 11$ \\
\hline \multicolumn{5}{|l|}{$B$-factors } \\
\hline Protein & 23.6 & 32.8 & 22.3 & $37.5 / 51.4$ \\
\hline lons $\left(\mathrm{PO}_{4}^{3-} / \mathrm{SO}_{4}{ }^{2-}\right)$ & - & $88.3 / 88.1$ & - & - \\
\hline Water/PEG & $41.7 /-$ & $49.6 /-$ & $32.1 / 23.6$ & $55.9 / 52.6$ \\
\hline \multicolumn{5}{|l|}{ R.m.s. deviations } \\
\hline Bond lengths $(\AA)$ & 0.011 & 0.012 & 0.010 & 0.016 \\
\hline Bond angles $\left({ }^{\circ}\right)$ & 1.666 & 1.728 & 1.668 & 1.900 \\
\hline
\end{tabular}

*Number of crystals for each structure: 1.

†Values in parenthesis are for the highest-resolution shell. 


\section{nature portfolio}

Corresponding author(s): Andres Palencia \& Malene Ringkjøbing Jensen

Last updated by author(s): Dec 22, 2021

\section{Reporting Summary}

Nature Portfolio wishes to improve the reproducibility of the work that we publish. This form provides structure for consistency and transparency

in reporting. For further information on Nature Portfolio policies, see our Editorial Policies and the Editorial Policy Checklist.

\section{Statistics}

For all statistical analyses, confirm that the following items are present in the figure legend, table legend, main text, or Methods section.

n/a Confirmed

\The exact sample size $(n)$ for each experimental group/condition, given as a discrete number and unit of measurement

X $\square$ A statement on whether measurements were taken from distinct samples or whether the same sample was measured repeatedly

$\triangle$ The statistical test(s) used AND whether they are one- or two-sided

Xnly common tests should be described solely by name; describe more complex techniques in the Methods section.

\ $\square$ A description of all covariates tested

$\square$ \A description of any assumptions or corrections, such as tests of normality and adjustment for multiple comparisons

$\square$ A full description of the statistical parameters including central tendency (e.g. means) or other basic estimates (e.g. regression coefficient)

AND variation (e.g. standard deviation) or associated estimates of uncertainty (e.g. confidence intervals)

Х For null hypothesis testing, the test statistic (e.g. $F, t, r$ ) with confidence intervals, effect sizes, degrees of freedom and $P$ value noted

Give $P$ values as exact values whenever suitable.

Х $\square$ For Bayesian analysis, information on the choice of priors and Markov chain Monte Carlo settings

Х $\square$ For hierarchical and complex designs, identification of the appropriate level for tests and full reporting of outcomes

Х $\square$ Estimates of effect sizes (e.g. Cohen's $d$, Pearson's $r$ ), indicating how they were calculated

Our web collection on statistics for biologists contains articles on many of the points above.

\section{Software and code}

Policy information about availability of computer code

Data collection NMR data acquisition: TopSpin version 3.5, VnmrJ version 3.1

X-ray data collection: MXCube version 3

Data analysis Analysis of NMR relaxation dispersion data: Open source software ChemEx version 2021.4.0-dev2 (https://github.com/gbouvignies/ChemEx) Pocket volume calculations: POVME version 3.0

Indexing and integration, X-ray crystallography data: XDS version 20200417, autoProc version 1.1.7

Data reduction, $\mathrm{X}$-ray crystallography: Pointless version 1.12.2, Aimless version 0.7.4

Molecular replacement, $X$-ray crystallography: Phaser version 2.8 .3

Model building and refinement, $X$-ray crystallography: Coot version 0.8.9.2, Refmac version 5.8.0258, CCP4I version 7.0.078 or 7.1.008

For manuscripts utilizing custom algorithms or software that are central to the research but not yet described in published literature, software must be made available to editors and reviewers. We strongly encourage code deposition in a community repository (e.g. GitHub). See the Nature Portfolio guidelines for submitting code \& software for further information.

\section{Data}

Policy information about availability of data

All manuscripts must include a data availability statement. This statement should provide the following information, where applicable:

- Accession codes, unique identifiers, or web links for publicly available datasets

- A description of any restrictions on data availability

- For clinical datasets or third party data, please ensure that the statement adheres to our policy 
SH3-H493A), 7NYM (JIP1-SH3-V517A), 7NZC (POSH-SH3-1) and 7NZD (POSH-SH3-4). The 1H, 13C and 15N chemical shifts of JIP1-SH3 have been deposited in the Biological Magnetic Resonance Data Bank with accession codes: 50814 (JIP1-SH3), 50817 (JIP1-SH3-Y526A), 50816 (JIP1-SH3-V517A), 50818 (JIP1-SH3-H493A), 50815 (JIP1-SH3-A541L).

SH3 domain structures for molecular replacement were retrieved from the PDB with accession codes: 2FPE (JIP1), 2LI1 (Sorbin and SH3 domain-containing protein 1) and 2SRC (tyrosine protein kinase C-Src). Structures for the proteome-wide SH3 sequence analysis were retrieved from the PDB with accession codes: 1CSK, 3A98, 209S, 5VEI and 4LNP.

\section{Field-specific reporting}

Please select the one below that is the best fit for your research. If you are not sure, read the appropriate sections before making your selection. $\bigotimes$ Life sciences Behavioural \& social sciences Ecological, evolutionary \& environmental sciences

For a reference copy of the document with all sections, see nature.com/documents/nr-reporting-summary-flat.pdf

\section{Life sciences study design}

All studies must disclose on these points even when the disclosure is negative.

Sample size
Data exclusions Datistical methods were not used to determine sample size.
Replication
Each crystal structure was solved from a single crystal. All nmr dispersion experiments were performed once with technical replicates as
described in the Methods section.
Blinding

\section{Reporting for specific materials, systems and methods}

We require information from authors about some types of materials, experimental systems and methods used in many studies. Here, indicate whether each material, system or method listed is relevant to your study. If you are not sure if a list item applies to your research, read the appropriate section before selecting a response.

\begin{tabular}{l} 
Materials \& experimental syste \\
\hline $\mathrm{n} / \mathrm{a}$ Involved in the study \\
$\square$ \\
$\square$ \\
$\square$ Antibodies \\
$\square$ Eukaryotic cell lines \\
$\square$ \\
$\square$ \\
$\square$ Palaeontology and archaeology \\
$\square$ \\
$\square$ Animals and other organisms \\
$\square$ Clinical data \\
$\square$ Dual use research of concern
\end{tabular}

\begin{tabular}{l} 
Methods \\
\hline n/a \\
$\square$ \\
$\square$
\end{tabular}

